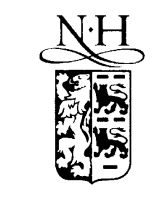

ELSEVIER

\title{
An investigation of the parametric X-rays along the velocity of emitting particle
}

\author{
A. Kubankin ${ }^{\text {a }}$, N. Nasonov ${ }^{\text {a,* }}$, V. Sergienko ${ }^{\text {b }}$, I. Vnukov ${ }^{\text {c }}$ \\ a Laboratory of Radiation Physics, Belgorod State University, 14 Studencheskaya Str., 308007 Belgorod, Russia \\ ${ }^{\mathrm{b}}$ Lebedev Physical Institute, Russian Academy of Science, 53 Leninsky Prospect, 117924 Moscow, Russia \\ ${ }^{\mathrm{c}}$ Nuclear Physics Institute at Tomsk Polytechnic University, Lenin Avenue 2a, P.O. Box 25, 634050 Tomsk, Russia
}

Received 14 March 2002; received in revised form 12 November 2002

\begin{abstract}
Parametric X-rays (PXR) along the velocity of relativistic electrons crossing a crystalline target is studied in this work. The detailed theory of PXR for Laue scattering geometry is developed with account of contributions of both PXR and transition radiation (TR) to the total emission yield. An influence of photoabsorption and interference between PXR and TR on the forward PXR properties are studied. Most appropriate conditions for the real experiment devoted to forward PXR observation are elucidated on the basis of the developed theory. The advantage of the use of heavy crystals with this aim in mind is shown. The expected experimental results are discussed.
\end{abstract}

(c) 2002 Elsevier Science B.V. All rights reserved.

PACS: 78.70.-g; 78.70. $\mathrm{C}_{k} ; 79.90 .+\mathrm{t}$

Keywords: Relativistic electron; X-ray emission; Dynamical diffraction

\section{Introduction}

When a fast charged particle moves in a crystal it can emit X-rays due to the coherent scattering of such a particle equilibrium electromagnetic field on periodically placed atomic planes of the crystal [1-3]. The theory of such emission known as the parametric X-rays or PXR predicts an existence of two PXR peaks propagating along both Bragg scattering direction and the velocity of an emitting

\footnotetext{
${ }^{*}$ Corresponding author. Tel.: +7-22-341477; fax: +7-22341692.

E-mail address: nnn@bsu.edu.ru (N. Nasonov).
}

particle. The first of mentioned peaks has been investigated theoretically and experimentally in detail [4-7]. Its theoretical description is based on both kinematical and dynamical theories of X-ray diffraction $[8,9]$. It is important that the influence of the density effect analogous to that in relativistic particle ionization energy losses hampers the manifestation of dynamical diffraction effects in such "ordinary PXR" [10]. Therefore kinematical and dynamical descriptions of PXR peak emitted to Bragg scattering direction are close to each other.

Additional PXR peak emitted along an emitting particle velocity is of the great physical interest because of two circumstances. First of all this peak 
can be described within the frame of dynamical diffraction theory only $[11,12]$. Therefore its observation will provide a convincing demonstration of the dynamical diffraction effects in PXR. Furthermore, the discussed PXR peak is interpreted usually as kind of Cherenkov effect appearing due to the dynamical changing of the effective refractive index of the crystal $[5,13]$. Verification of such $\mathrm{X}$-ray Cherenkov radiation is very difficult experimental task. Some attempts at "forward PXR" observation have not been successful $[14,15]$. It seems likely that this radiation has been observed for the first time by Mainz group [16], where a 50 $\mu \mathrm{m}$ silicon crystal and $855 \mathrm{MeV}$ electron beam of the Mainz Microtron MAMI have been used. It should be noted that the peak [16] was observed within the wide region of observation angles, but the nature of such a peak observed at small angles relative to emitting particle velocity $\Theta \sim \gamma^{-1}=m / \epsilon$ ( $m$ and $\epsilon$ are the mass and the energy of emitting electron) can be connected with the dynamical diffraction effects in the transition radiation (TR) from a thin crystalline target [17]. There is no detailed description of the experiment [16] in literature as well as its theoretical explanation. Therefore the detailed theoretical description of the forward PXR from a crystalline target with the finite thickness $L$ is one of the currently central problems of PXR theory.

Forward PXR properties were analyzed theoretically in several works [11-13] (see first of all the last work [13]), but the most part of obtained in these works analytical and numerical results describe PXR contribution only. However it is necessary to take into account the TR contribution to emission yield because a total TR yield is greater than PXR one. Since PXR spectral width is very small compared with TR width the measurements of differential characteristics of the emission are needed for the separation of PXR on the TR background. It should be noted that TR properties can be changed very essentially in the vicinity of Bragg frequency where the forward PXR is realized [17], therefore the study of TR contribution and an interference between TR and PXR is very important for the correct description of real experiment.

The forward PXR in Laue scattering geometry is considered in this work on the basis of dynam- ical diffraction theory. The main goal of the developed theory consists in the elucidation of most appropriate conditions for the forward PXR experimental verification. In Section 2 the general expression for an emission spectral-angular distribution is derived on the basis of the previous work [18]. Obtained results are used in Section 3 for the detailed analysis of emission characteristics. Section 4 is devoted to the discussion of experimental conditions. Our conclusions and some final comments are collected in Section 5.

\section{General expressions}

Let us consider an emission of relativistic electrons crossing a crystal with the thickness $L$ along the axis $\mathbf{e}$ as it is shown in Fig. 1. This axis is usually fixed in an experimental setup. The axis OX in Fig. 1 is the normal to the surface of the crystal target. This axis coincides with the travelling axis of the goniometer in which the target is arranged. The stationary axis of the goniometer coincides with the axis OZ in Fig. 1. The angles $\theta_{\|}^{\prime}$ and $\theta_{\perp}^{\prime}$ in Fig. 1 show the possible turning of the crystalline target around the corresponding axes. Reflecting crystallographic plane of the crystal

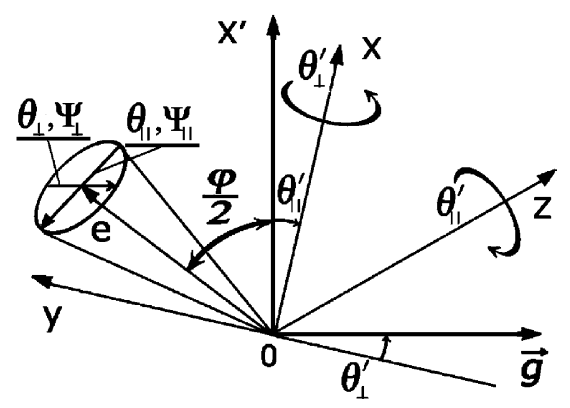

Fig. 1. Geometry of the forward PXR. An emitting electron beam and emitted photon flux are directed along the axis e. The axis $\mathrm{OX}$ is the travelling axis of the goniometer. The axis $\mathrm{OZ}$ is the stationary axis of the goniometer. The reflecting crystallographic plane go through the axis OX perpendicular to reciprocal lattice vector $\mathbf{g}$. The axis $\mathrm{OX}^{\prime}$ is the initial position of the axis $\mathrm{OX}$ (before possible turning by the goniometer). The angle $\varphi / 2$ is initial angle between electron beam axis and reflecting plane. Two-dimensional angular variables $\Theta$ and $\Psi$ describe angular distributions of the photon flux and electron beam respectively. 
determined by the reciprocal lattice vector $\mathbf{g}$ (see Fig. 1) is arranged parallel to the axis OX. The angle $\varphi / 2$ is the initial orientation angle (when $\theta_{\|}^{\prime}=\theta_{\perp}^{\prime}=0$ ) between an electron beam axis and the reflecting plane. Characteristics of the photon flux, emitted along the axis $\mathbf{e}$ and propagating in a vacuum behind the crystal are analyzed in this work. Such task was considered earlier in work [18], but for the special case $\theta_{\|}^{\prime}=\theta_{\perp}^{\prime}=0$ only. Since the aim of our analysis is to elucidate the experimental possibilities to separate PXR contribution on the background of TR more general expression for the total emission amplitude $\mathbf{A}$ compared to given in [18] is derived here.

Describing the periodically changing dielectric permeability of the crystal $\epsilon(\omega, \mathbf{r})$ as

$\epsilon(\omega, \mathbf{r})=1+\chi_{0}(\omega)+\sum_{\mathbf{g}}^{\prime} \chi_{\mathbf{g}}(\omega) \mathrm{e}^{\mathrm{igr}}$

and defining the Fourier transform of the excited transverse electric field $\mathbf{E}_{\omega}^{\mathrm{tr}}(\mathbf{r})$ within the frame of well known two-wave approximation of dynamical diffraction theory [19] as

$\mathbf{E}_{\omega}^{\mathrm{tr}}=\int \mathrm{d}^{3} k \sum_{\lambda=1}^{2}\left(\mathbf{e}_{\lambda 0} E_{\lambda 0} \mathrm{e}^{\mathrm{i} \mathbf{k r}}+\mathbf{e}_{\lambda \mathbf{g}} E_{\lambda \mathbf{g}} \mathrm{e}^{\mathrm{i}(\mathbf{k}+\mathbf{g}) \mathbf{r}}\right)$,

where $\mathbf{e}_{\lambda 0}$ and $\mathbf{e}_{\lambda \mathrm{g}}$ are the polarization vectors, $\mathbf{k e}_{\lambda 0}=(\mathbf{k}+\mathbf{g}) \mathbf{e}_{\lambda \mathbf{g}}=0$ one can obtain from Maxwell equations the ordinary for PXR theory system

$$
\begin{aligned}
& \left(k^{2}-\omega^{2}\left(1+\chi_{0}\right)\right) E_{\lambda 0}-\omega^{2} \chi_{-\mathbf{g}} \alpha_{\lambda} E_{\lambda \mathbf{g}} \\
& \quad=\frac{\mathrm{i} \omega e}{2 \pi^{2}} \mathbf{e}_{\lambda 0} \mathbf{v} \delta(\omega-\mathbf{k v}), \\
& \left((\mathbf{k}+\mathbf{g})^{2}-\omega^{2}\left(1+\chi_{0}\right)\right) E_{\lambda \mathbf{g}}-\omega^{2} \chi_{\mathbf{g}} \alpha_{\lambda} E_{\lambda 0}=0,
\end{aligned}
$$

where $\mathbf{v}$ is the emitting electron velocity, $\alpha_{1}=1$, $\alpha_{2}=\mathbf{e}_{20} \mathbf{e}_{2 \mathrm{~g}}=\cos \varphi$.

As mentioned in introduction, the discussed forward PXR is interpreted as Cherenkov like effect. Since such an opinion is not the sole it should present an added reason for utilizing quasi-Cherenkov nature of the forward PXR following from (1). When a fast electron moves in an unbounded crystal the solution $E_{\lambda 0}$ can be presented in the form

$$
\begin{aligned}
& E_{\lambda 0}=\frac{i \omega e}{2 \pi^{2}} \frac{\mathbf{e}_{\lambda 0} \mathbf{v}}{k^{2}-\omega^{2} \epsilon_{\mathrm{eff}}(\omega, \mathbf{k})} \delta(\omega-\mathbf{k v}), \\
& \epsilon_{\mathrm{eff}}=1+\chi_{0}+\frac{\omega^{2} \chi_{\mathrm{g}} \chi_{-\mathbf{g}} \alpha_{\lambda}^{2}}{(\mathbf{k}+\mathbf{g})^{2}-\omega^{2}\left(1+\chi_{0}\right)},
\end{aligned}
$$

coinciding formally with that describing an electromagnetic field excited by a fast particle in a homogeneous medium with the spatial dispersion. The presented expression for the effective dielectric permeability $\epsilon_{\text {eff }}$ shows clearly that the possible emission has the quasi-Cherenkov nature and this emission can occur due to dynamical changing of the refractive index only, since $\chi_{0}(\omega) \approx-\omega_{0}^{2} / \omega^{2}<$ 0 in X-ray range.

On the other hand the solution $E_{\lambda \mathrm{g}}$ cannot be represented in the form, analogous to (2). In accordance with (1b) the expression for $E_{\lambda \mathrm{g}}$ has the form

$$
\begin{aligned}
E_{\lambda \mathbf{g}}= & \frac{i \omega e}{2 \pi^{2}} \frac{\mathbf{e}_{\lambda 0} \mathbf{v}}{k^{2}-\omega^{2} \epsilon_{\mathrm{eff}}} \\
& \times \frac{\omega^{2} \chi_{\mathbf{g}} \alpha_{\lambda}}{k_{\mathbf{g}}^{2}-\omega^{2}\left(1+\chi_{0}\right)} \delta\left(\omega-\mathbf{k}_{\mathbf{g}} \mathbf{v}+\mathbf{g v}\right),
\end{aligned}
$$

where we take into account that the momentum $\mathbf{k}_{\mathbf{g}} \equiv \mathbf{k}+\mathbf{g}$ corresponds to the field $E_{\lambda \mathbf{g}}$, propagating along the Bragg scattering direction. Obviously, the field $E_{\lambda \mathrm{g}}$ appears due to the coherent scattering of the field $E_{\lambda 0}$ by a system of parallel atomic planes, determined by the reciprocal lattice vector $\mathbf{g}$ (the scattering process is indicated by the momentum transfer $-\mathbf{g}$ in the kinematical conservation low $\left.\omega-\mathbf{k}_{\mathbf{g}} \mathbf{v}=-\mathbf{g v}\right)$. It is easy to see that the emission field $E_{\lambda \mathrm{g}}$ occurs independently on the character of the primary field $E_{\lambda 0}$. Particularly, the discussed emission occurs without account of the dynamical diffraction effects, when $\epsilon_{\text {eff }} \rightarrow 1+\chi_{0}$ and the field $E_{\lambda 0}$ is reduced to ordinary coulomb field of a fast particle. This approximation is known in PXR theory as the kinematical theory of PXR $[1,8]$.

Returning to the general expressions (1) let us consider an emission from the finite crystal target. Using the general solutions of both these equations and corresponding equations for fields in a vacuum outside the crystal (such equations follow from (1) in the limit $\chi_{0}=\chi_{\mathrm{g}}=\chi_{-\mathrm{g}}=0$ ) one can 
obtain by the use of ordinary boundary conditions for the fields at the in and out-surfaces of the crystal the following expression for the radiation field in a vacuum behind the crystal:

$E_{\lambda 0}^{\mathrm{Rad}}=a_{\lambda \mathbf{k}_{\|}} \delta\left(k_{x}-p\right)$,

$$
\begin{aligned}
a_{\lambda \mathbf{k}_{\|}}=- & \frac{\mathrm{i} \omega e}{2 \pi^{2}} \mathbf{e}_{\lambda 0} \mathbf{v}\left[\left(1+\frac{\Delta}{\sqrt{\Delta^{2}+\omega^{4} \chi_{\mathrm{g}} \chi_{-\mathrm{g}} \alpha_{\lambda}^{2}}}\right)\right. \\
& \times\left(\frac{1}{k_{*}^{2}-p^{2}}-\frac{1}{k_{*}^{2}-k_{1}^{2}}\right)\left(1-\mathrm{e}^{-\mathrm{i}\left(k_{*}-k_{1}\right) L}\right) \\
& +\left(1-\frac{\Delta}{\sqrt{\Delta^{2}+\omega^{4} \chi_{\mathrm{g}} \chi_{-\mathrm{g}} \alpha_{\lambda}^{2}}}\right) \\
& \left.\times\left(\frac{1}{k_{*}^{2}-p^{2}}-\frac{1}{k_{*}^{2}-k_{2}^{2}}\right)\left(1-\mathrm{e}^{-\mathrm{i}\left(k_{*}-k_{2}\right) L}\right)\right],
\end{aligned}
$$

where the following designations are used:

$$
\begin{aligned}
& p=\sqrt{\omega^{2}-k_{\|}^{2}}, \quad \Delta=\frac{1}{2}\left(g^{2}+2 \mathbf{k}_{\|} \mathbf{g}\right), \\
& k_{1,2}^{2}=p^{2}+\omega^{2} \chi_{0}-\Delta \pm \sqrt{\Delta^{2}+\omega^{4} \chi_{\mathrm{g}} \chi_{-\mathrm{g}} \alpha_{\lambda}^{2}}, \\
& k_{*}=\frac{1}{v_{x}}\left(\omega-\mathbf{k}_{\|} \mathbf{v}_{\|}\right) .
\end{aligned}
$$

Here $\mathbf{v}_{\|}$and $\mathbf{k}_{\|}$are the components of corresponding vectors perpendicular to the axis $\mathrm{OX}$ in Fig. 1. Fourier transform of the emission field $\mathbf{E}_{\omega}^{\mathrm{Rad}}(\mathbf{r})$ in wave-zone is determined by the integral $E_{\omega \lambda}^{\mathrm{Rad}}=\int \mathrm{d}^{3} k \mathrm{e}^{\mathrm{ikr}} E_{\lambda 0}^{\mathrm{Rad}} \rightarrow A_{\lambda} \frac{\mathrm{e}^{\mathrm{i} \omega r}}{r}$,

$A_{\lambda}=-2 \pi \mathrm{i} \omega n_{x} a_{\lambda \omega \mathbf{n}_{\|}}$,

calculated by the stationary phase method. Here $\mathbf{n}=\mathbf{n}_{\|}+n_{x} \mathbf{e}_{x}$ is the unit vector to the direction of emitted photon observation.

To describe an influence of both emitting electron multiple scattering and initial angular spread of the beam on the angular distribution of emitted photons it is convenient to introduce two-dimensional angular variables $\Psi$ and $\Theta$ by the formulae

$$
\begin{aligned}
& \mathbf{v}=\mathbf{e}\left(1-\frac{1}{2} \gamma^{-2}-\frac{1}{2} \Psi^{2}\right)+\Psi, \quad \mathbf{e} \Psi=0, \\
& \mathbf{n}=\mathbf{e}\left(1-\frac{1}{2} \Theta^{2}\right)+\Theta, \quad \mathbf{e} \Theta=0,
\end{aligned}
$$

where $\gamma$ is the Lorentz factor. Using (7) and simplest approximation for the crystal dielectric susceptibilities

$\chi_{0}=-\frac{\omega_{0}^{2}}{\omega^{2}}+\mathrm{i} \chi_{0}^{\prime \prime}, \quad \chi_{\mathbf{g}}=\chi_{-\mathbf{g}}=-\frac{\omega_{\mathbf{g}}^{2}}{\omega^{2}}+\mathrm{i} \chi_{\mathbf{g}}^{\prime \prime}$,

one can represent the expression for emission amplitude $A_{\lambda}$ in the final form

$$
\begin{aligned}
A_{\lambda}=A_{\lambda}^{\mathrm{TR}} & +A_{\lambda}^{\mathrm{PXR}}, \\
A_{\lambda}^{\mathrm{TR}}=- & \frac{e}{2 \pi} \mathbf{e}_{\lambda 0} \mathbf{v}\left(\frac{1}{\Omega_{0}}-\frac{1}{\Omega}\right)\left[\left(1+\frac{\tau}{\sqrt{\tau^{2}+\alpha_{\lambda}^{2}}}\right)\right. \\
& \times\left(1-\exp \left(-\frac{\mathrm{i} \omega L}{2 \cos (\varphi / 2)}\left(\sigma_{-}^{\prime}-\mathrm{i} \sigma_{-}^{\prime \prime}\right)\right)\right) \\
& +\left(1-\frac{\tau}{\sqrt{\tau^{2}+\alpha_{\lambda}^{2}}}\right) \\
& \left.\times\left(1-\exp \left(-\frac{\mathrm{i} \omega L}{2 \cos (\varphi / 2)}\left(\sigma_{+}^{\prime}-\mathrm{i} \sigma_{+}^{\prime \prime}\right)\right)\right)\right] \\
A_{\lambda}^{\mathrm{PXR}}= & \frac{e}{2 \pi} \frac{\mathbf{e}_{\lambda 0} \mathbf{v}}{\sqrt{\tau^{2}+\alpha_{\lambda}^{2}}} \frac{\omega_{\mathbf{g}}^{2} \alpha_{\lambda}^{2}}{\omega^{2} \Omega}\left[\frac{1}{\sigma_{-}^{\prime}-\mathrm{i} \sigma_{-}^{\prime \prime}}\right. \\
& \times\left(1-\exp \left(-\frac{\mathrm{i} \omega L}{2 \cos (\varphi / 2)}\left(\sigma_{-}^{\prime}-\mathrm{i} \sigma_{-}^{\prime \prime}\right)\right)\right) \\
& -\frac{1}{\sigma_{+}^{\prime}-\mathrm{i} \sigma_{+}^{\prime \prime}}\left(1-\exp \left(-\frac{\mathrm{i} \omega L}{2 \cos (\varphi / 2)}\right.\right. \\
& \left.\left.\left.\times\left(\sigma_{+}^{\prime}-\mathrm{i} \sigma_{+}^{\prime \prime}\right)\right)\right)\right],
\end{aligned}
$$

where

$$
\begin{aligned}
& \Omega_{0}=\gamma^{-2}+\left(\Theta_{\perp}-\Psi_{\perp}\right)^{2}+\left(\Theta_{\|}-\Psi_{\|}\right)^{2}, \\
& \Omega=\Omega_{0}+\frac{\omega_{0}^{2}}{\omega^{2}}, \quad \tau=\frac{g^{2}}{2 \omega_{\mathbf{g}}^{2}}\left(1-\frac{\omega}{\omega_{\mathrm{B}}^{\prime}}\right), \\
& \omega_{\mathrm{B}}^{\prime}=\omega_{\mathrm{B}}\left(1+\left(\Theta_{\|}^{\prime}+\Theta_{\|}\right) \cot \frac{\varphi}{2}\right)^{-1}, \\
& \omega_{\mathrm{B}}=g / 2 \sin (\varphi / 2),
\end{aligned}
$$


$\mathbf{e}_{10} \mathbf{v}=\Theta_{\perp}-\Psi_{\perp}, \quad \mathbf{e}_{20} \mathbf{v}=\Theta_{\|}-\Psi_{\|}$,

$\sigma_{ \pm}^{\prime}=\Omega+\frac{\omega_{\mathbf{g}}^{2}}{\omega^{2}}\left(\tau \pm \sqrt{\tau^{2}+\alpha_{\lambda}^{2}}\right)$

$\sigma_{ \pm}^{\prime \prime}=\chi_{0}^{\prime \prime} \pm \chi_{\mathrm{g}}^{\prime \prime} \frac{\alpha_{\lambda}^{2}}{\sqrt{\tau^{2}+\alpha_{\lambda}^{2}}}$.

Here $\omega_{0}$ is the plasma frequency, $\omega_{\mathbf{g}}^{2}=$ $\omega_{0}^{2}(F(g) / Z)(S(\mathbf{g}) / N) \mathrm{e}^{-\frac{1}{2} g^{2} u^{2}}, F(g) \quad$ is the atom formfactor, $Z$ is the number of electrons in an atom, $S(\mathbf{g})$ is the structure factor of a crystal elementary cell containing $N$ atoms, $u$ is the meansquare amplitude of atomic thermal vibrations, the directions of $\Theta$ and $\Psi$ component variations are shown in Fig. 1.

Presented formulae (9), where the total emission amplitude $A_{\lambda}$ is given as a sum of TR and PXR amplitudes, are very convenient for the further analysis of the possibilities to separate PXR contribution on TR background.

\section{Properties of the forward PXR and TR}

Formulae (9) and (10) allow to search a separate PXR contribution to total emission yield, TR background and an interference between these emission mechanisms versus some important parameters determining the experimental conditions of PXR observation.

First of all let us consider the separate PXR contribution. In accordance with (9c) two branches of possible X-ray waves in a crystal determine the structure of PXR spectral-angular distribution, but only one of them corresponding to signe (-) makes essential contribution because only the quantity $\sigma_{-}^{\prime}$ can be equal to zero in the corresponding denominator in the expression for $A_{\lambda}^{\mathrm{PXR}}$. Relatively simple formula for PXR spectral-angular distribution

$$
\begin{aligned}
& \omega \frac{\mathrm{d} N_{\lambda}^{\mathrm{PXR}}}{\mathrm{d} \omega \mathrm{d}^{2} \Theta}=\frac{e^{2}}{4 \pi^{2}}\left\langle\frac{\left(\mathbf{e}_{\lambda 0} \mathbf{v}\right)^{2}}{\tau^{2}+\alpha_{\lambda}^{2}} \frac{\omega_{\mathbf{g}}^{4} \alpha_{\lambda}^{4}}{\omega^{4} \Omega^{2}}\right. \\
&\left.\times \frac{1+\mathrm{e}^{-\frac{\omega L}{\cos (\varphi / 2) \sigma_{-}^{\prime \prime}}}-2 \mathrm{e}^{-\frac{\omega L}{\cos (\phi / 2) \sigma_{-}^{\prime \prime}}} \cos \left(\frac{\omega L}{\cos (\varphi / 2)} \sigma_{-}^{\prime}\right)}{\left(\sigma_{-}^{\prime}\right)^{2}+\left(\sigma_{-}^{\prime \prime}\right)^{2}}\right\rangle,
\end{aligned}
$$

follows from (9c) with account of such a circumstance. Here the brackets \langle\rangle mean the averaging over angles $\Psi_{\|}$and $\Psi_{\perp}$, determining the angular spread in an electron beam.

Presented formula (11) is very convenient for the general analysis of emission properties. Since a narrow PXR peak is located near to the Bragg frequency $\omega_{\mathrm{B}}$ (see (10)) [1] the vicinity of $\omega_{\mathrm{B}}$ is of interest for the purpose of our analysis. Therefore the "fast spectral variable" $\tau(\omega)$ is defined in (10) and (11) instead of the photon energy $\omega$ (one can see that small variations in $\omega$ corresponds to great variations in $\tau(\omega)$ because of large value of the coefficient $\left.g^{2} / 2 \omega_{\mathrm{g}}^{2} \gg 1\right)$. With account of this circumstance the quantity $\omega$ is assumed to be equal to $\omega_{\mathrm{B}}$ in the formula (11) exclusive of the function $\tau(\omega)$.

Formulae, analogous to (9)-(11) were used in work [17] for the analysis of an influence of dynamical diffraction effects on TR properties. The forward PXR contribution was considered as a background in this work. In contrast with that TR contribution is considered as a background in our work, therefore our study is directed to the determination of the conditions when TR contribution can be small enough.

Returning to the general result (11) let us consider PXR angular distribution following from (11) after integration over $\omega$. Since $\sigma_{-}^{\prime \prime} \ll 1$ such integration can be performed by the use of the approximation

$$
\begin{aligned}
& \frac{1}{x^{2}+\alpha^{2}}\left(1+\mathrm{e}^{-2 \beta \alpha}-2 \mathrm{e}^{-\beta \alpha} \cos (\beta x)\right) \\
& \rightarrow \frac{\pi}{\alpha}\left(1-\mathrm{e}^{-2 \beta \alpha}\right) \delta(x) .
\end{aligned}
$$

The result of integration has the form

$$
\begin{aligned}
\frac{\mathrm{d} N_{\lambda}^{\text {PXR }}}{\mathrm{d}^{2} \Theta}= & \frac{e^{2} \omega_{0}^{2}}{\pi g^{2}} \\
& \times \frac{\delta_{\lambda}^{4}}{\gamma_{*}^{6} \chi_{0}^{\prime \prime}}\left\langle\frac{\left(\mathbf{e}_{\lambda 0} \mathbf{v}\right)^{2}\left(1-\mathrm{e}^{-\frac{\omega L}{\cos (\varphi / 2)} \sigma_{-}^{\prime \prime}}\right)}{\Omega^{2}\left(\Omega^{2}+\gamma_{*}^{-4} \delta_{\lambda}^{2}-2 \kappa_{\lambda} \gamma_{*}^{-2} \delta_{\lambda} \Omega\right)}\right\rangle,
\end{aligned}
$$

where

$$
\begin{aligned}
& \gamma_{*}=\frac{\omega_{\mathrm{B}}}{\omega_{0}}=\frac{g}{2 \omega_{0} \sin (\varphi / 2)}, \quad \delta_{\lambda}=\frac{\omega_{\mathbf{g}}^{2}}{\omega_{0}^{2}} \alpha_{\lambda}, \\
& \kappa_{\lambda}=\frac{\chi_{\mathbf{g}}^{\prime \prime}}{\chi_{0}^{\prime \prime}} \alpha_{\lambda},
\end{aligned}
$$


$\Omega=\gamma^{-2}+\gamma_{*}^{-2}+(\Theta-\Psi)^{2}$,

$\sigma_{-}^{\prime \prime}=\chi_{0}^{\prime \prime}\left(1-\frac{2 \kappa_{\lambda} \gamma_{*}^{-2} \delta_{\lambda} \Omega}{\Omega^{2}+\gamma_{*}^{-4} \delta_{\lambda}^{2}}\right)$.

The physical meaning of introduced by (14) parameters $\gamma_{*}, \delta_{\lambda}$ and $\kappa_{\lambda}$ is clear. The parameter $\gamma_{*}$ describes an influence of the density effect in PXR [10]. Indeed, the spectrum of virtual photons associated with relativistic particle moving through a dense medium is suppressed in the frequency range $\omega<\gamma \omega_{0}$ [1]. Since only virtual photons with energies $\omega$ close to the Bragg frequency $\omega_{\mathrm{B}}$ make an essential contribution to the yield of PXR process this condition can be represented as $\omega_{\mathrm{B}} / \omega_{0}=$ $\gamma_{*}<\gamma$. On the other hand the ratio $\gamma / \gamma_{*}$ determines the role of dynamical diffraction effects in PXR. The forward PXR occurs due to dynamical changing of the effective dielectric permeability $\epsilon_{\text {eff }}$ in (2), which can be represented in the form

$$
\begin{aligned}
\epsilon_{\mathrm{eff}} \approx & 1-\frac{\omega_{0}^{2}}{\omega^{2}} \\
& \times\left(1-\delta_{\lambda}^{4} \frac{\gamma_{*}^{-2}}{\gamma^{-2}+\gamma_{*}^{-2}+(\Theta-\Psi)^{2}+2 \Delta / \omega_{\mathrm{B}}^{2}}\right) .
\end{aligned}
$$

Since $\delta_{\lambda} \leqslant 1$ the additional term in (15), caused by dynamical effects, can be essential on condition $\gamma \gg \gamma_{*}$ only.

Parameter $\kappa_{\lambda}$ describes an influence of the Borrmann effect (or the effect of anomalous photoabsorption) on the forward PXR. This parameter determines a possible decreasing of the effective absorption coefficient $\omega \sigma_{-}^{\prime \prime}$, defined by the corresponding formula in (14). Obviously, the necessary condition for the Borrmann effect manifestation in PXR $\kappa_{\lambda} \approx 1$ coincides with that in the physics of free X-ray scattering in a crystal [19].

In accordance with the determination of $\omega_{\mathbf{g}}^{2}$, given below the formulae (10), the coefficient $\delta_{\lambda}$ describes quantitatively the degree of non-uniformity in the crystal electron density caused by the fixed crystallographic plane.

It should be noted that the forward PXR angular distribution (13) does not depend on the orientation angles $\Theta_{\|}^{\prime}$ and $\Theta_{\perp}^{\prime}$. This property can be reasonably expected since the forward PXR photons are emitted along the fast electron velocity without regard to the relative position of an emitting particle and reflecting crystallographic plane.

Let us consider the result (13) in the limit of very thin target when the electron path in the target $L / \cos (\varphi / 2)$ is smaller than the effective absorption length $1 / \omega \sigma_{-}^{\prime \prime}$. The simple formula

$$
\frac{\mathrm{d} N_{\lambda}^{\mathrm{PXR}}}{\mathrm{d}^{2} \Theta}=\frac{e^{2} \omega_{0}^{2}}{\pi g^{2}} \frac{\omega_{\mathrm{B}} L}{\cos (\varphi / 2)} \frac{\delta_{\lambda}^{4}}{\gamma_{*}^{6}}\left\langle\frac{\left(\mathbf{e}_{\lambda} \mathbf{v}\right)^{2}}{\Omega^{2}\left(\Omega^{2}+\gamma_{*}^{-4} \delta_{\lambda}^{2}\right)}\right\rangle,
$$

following under these conditions from (13), coincides with the corresponding result [13].

Derived formula shows a very strong dependence of the forward PXR angular distribution on the emitting particle energy $\epsilon=\gamma m$. In accordance with (16) PXR angular density in the vicinity of its maximum is proportional to the coefficient $\left(1+\gamma_{*}^{2} / \gamma^{2}\right)^{-3}$, therefore $\left(\mathrm{d} N_{\lambda}^{\text {PXR }} / \mathrm{d}^{2} \Theta\right) \sim \gamma^{6} / \gamma_{*}^{6} \ll 1$ within the range of small particle energies $\epsilon<\gamma_{*} m$. Thus, the forward PXR can be observed with the understanding that

$\epsilon \gg \gamma_{*} m$

only, when the distribution (10) does not depend on $\gamma$.

It should be noted that the maximum of the angular distribution of PXR photons, emitted to Bragg scattering direction, is proportional to the coefficient $\left(1+\gamma_{*}^{2} / \gamma^{2}\right)^{-1}$, that is the $\gamma$-dependence of the forward PXR is significantly stronger than that of ordinary PXR.

Evidently, the distribution (16) decreases proportional to $\Theta^{-6}$ in the range of great values of observation angles $\Theta>\sqrt{\gamma^{-2}+\gamma_{*}^{-2}}$ [13], but more interesting property of this distribution consists in the shift of its maximum to the side of small observation angles compared to the angular distribution of ordinary PXR. Indeed, the maximum of the distribution (16) is located near to $\Theta \approx$ $(1 / \sqrt{3}) \sqrt{\gamma^{-2}+\gamma_{*}^{-2}}$ in contrast with that for the angular maximum of ordinary PXR located near to $\Theta \approx \sqrt{\gamma^{-2}+\gamma_{*}^{-2}}$.

Formula (13) predicts the possibility of the Borrmann effect manifestation in the forward PXR in the alternative limiting case of thick target, 
when $L / \cos (\varphi / 2) \gg 1 / \omega \sigma_{-}^{\prime \prime}$ and the result (13) is reduced to

$$
\begin{aligned}
\frac{\mathrm{d} N_{\lambda}^{\text {PXR }}}{\mathrm{d}^{2} \Theta}= & \frac{e^{2} \omega_{0}^{2}}{\pi g^{2}} \frac{1}{\chi_{0}^{\prime \prime}} \frac{\delta_{\lambda}^{4}}{\gamma_{*}^{6}} \\
& \times\left\langle\frac{\left(\mathbf{e}_{\lambda 0} \mathbf{v}\right)^{2}}{\Omega^{2}\left(\Omega^{2}+\gamma_{*}^{-4} \delta_{\lambda}^{2}-2 \kappa_{\lambda} \gamma_{*}^{-2} \delta_{\lambda} \Omega\right)}\right\rangle .
\end{aligned}
$$

In accordance with (18) such an effect can be realized for high energy particles $\left(\epsilon \gg \gamma_{*} m\right)$ and highly reflecting crystallographic planes $\left(\delta_{\lambda} \approx 1\right)$ only. The forward PXR angular distribution, calculated by (18) for fixed values of the parameters $\delta_{\lambda}, \gamma / \gamma_{*}$ and different values of the parameter $\kappa_{\lambda}$, is illustrated by curves presented in Fig. 2. These curves demonstrate a shift of the maximum of PXR angular distribution to the side of small observation angles and essential growth of its amplitude when increasing of the parameter $\kappa_{\lambda}$. The experimental verification of the Borrmann effect in PXR is a difficult task because of the necessity to satisfy the many conditions. It seems the best way to observe this effect is to choose the Bragg frequency $\omega_{\mathrm{B}}$ in the frequency range where such an effect has been observed earlier in the process of free X-ray scattering in given crystal.

Let us consider now an influence of multiple scattering and initial angular spread of electron

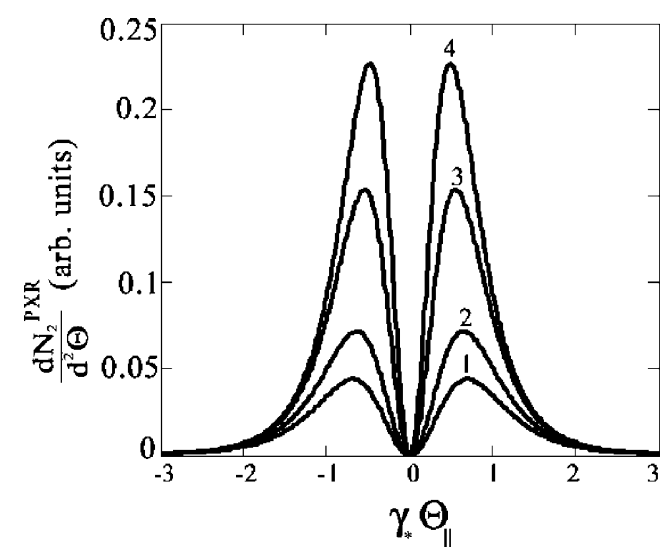

Fig. 2. The Borrmann effect manifestation in the forward PXR. Presented curves have been calculated for fixed values of the parameters $\gamma / \gamma_{*}=2, \delta_{2}=0.8$ and different values of the parameter $\kappa_{2}: 1-\kappa_{2}=0 ; 2-\kappa_{2}=0.5 ; 3-\kappa_{2}=0.9 ; 4-\kappa_{2}=1$. beam on PXR angular distribution. In condition $L / \cos (\varphi / 2) \gg 1 / \omega \sigma_{-}^{\prime \prime}$ under consideration PXR yield is formed at a small part of the electron path in the target. Therefore one can use the electron distribution function $f(\Psi, L / \cos (\varphi / 2))$ at the output of the target to perform the averaging over $\Psi$ in PXR angular distribution (18). Assuming that $f(\Psi, L / \cos (\varphi / 2))=\left(1 / \pi \Psi_{\mathrm{L}}^{2}\right) \exp \left(-\Psi^{2} / \Psi_{\mathrm{L}}^{2}\right)$, $\Psi_{\mathrm{L}}^{2}=\Psi_{0}^{2}+\Psi_{\mathrm{S}}^{2}, \Psi_{0}$ is the initial beam spread, $\Psi_{\mathrm{S}}^{2}=\epsilon_{k}^{2} L / \epsilon^{2} L_{\mathrm{R}} \cos (\varphi / 2), \epsilon_{k} \approx 21 \mathrm{MeV}, L_{\mathrm{R}}$ is the radiation length, one can obtain from (18) with the proviso that $\epsilon \gg \gamma_{*} m, \kappa_{\lambda} \ll 1, \delta_{\lambda} \ll 1$ the following expression

$$
\begin{aligned}
\frac{\mathrm{d} N_{\lambda}^{\mathrm{PXR}}}{\mathrm{d}^{2} \Theta}=\frac{e^{2} \omega_{0}^{2}}{2 \pi g^{2}} \frac{\delta_{\lambda}^{4}}{\chi_{0}^{\prime \prime}} \int_{0}^{\infty} \frac{\mathrm{d} t \mathrm{e}^{-t}}{\left(a^{2}-4 \gamma_{*}^{2} \Theta^{2} \gamma_{*}^{2} \Psi_{\mathrm{L}}^{2} t\right)^{\frac{7}{2}}}\left[2 \gamma_{*}^{2} \Theta_{\lambda}^{2} a^{3}\right. \\
+\left(a^{3}-4 a^{2} \gamma_{*}^{2} \Theta_{\lambda}^{2}-12 a \gamma_{*}^{2} \Theta_{\lambda}^{2}-16 \gamma_{*}^{2} \Theta_{\lambda}^{2} \gamma_{*}^{2} \Psi_{\mathrm{L}}^{2} t\right. \\
\left.\left.\quad+4 a\left(2 \gamma_{*}^{2} \Theta_{\lambda}^{2}-\gamma_{*}^{2} \Theta^{2}\right) \gamma_{*}^{2} \Psi_{\mathrm{L}}^{2} t\right) \gamma_{*}^{2} \Psi_{\mathrm{L}}^{2} t\right]
\end{aligned}
$$

where $a=1+\gamma_{*}^{2} \Theta^{2}+\gamma_{*}^{2} \Psi_{\mathrm{L}}^{2} t$. In accordance with (19) effective values of the variable $t$ is about $t_{\text {eff }} \approx 1$, therefore an influence of multiple scattering on PXR angular distribution is determined by the parameter $\gamma_{*} \Psi_{\mathrm{L}}$. One can neglect the multiple scattering with the understanding that

$\Psi_{\mathrm{L}} \ll \gamma_{*}^{-1}$.

When this requirement is compared with that for TR $\Psi_{\mathrm{L}} \ll \gamma^{-1}$, it is apparent that PXR angular distribution is considerably less sensitive to multiple scattering because PXR angular scale $\Delta \Theta \sim$ $\gamma_{*}^{-1}$ is many times the TR scale $\Delta \Theta \sim \gamma^{-1}$ on condition (17) when PXR contribution can be essential. An influence of the multiple scattering on the forward PXR angular distribution is illustrated by the curves presented in Fig. 3 calculated for different values of the parameter $\gamma_{*} \Psi_{\mathrm{L}}$.

Returning to the general formula (11) let us consider the forward PXR spectral distribution. The formula

$$
\begin{aligned}
\frac{\mathrm{d} N_{\lambda}^{\text {PXR }}}{\mathrm{d} \omega}= & \frac{e^{2} \omega_{0}^{2}}{\pi g^{2}} \frac{\delta_{\lambda}^{4}}{\gamma_{*}^{6} \chi_{0}^{\prime \prime}} \int \mathrm{d}^{2} \Theta \\
& \times\left\langle\frac{\left(\mathbf{e}_{\lambda 0} \mathbf{v}\right)^{2}\left[1-\exp \left(-\frac{\omega L}{\cos (\varphi / 2)} \sigma_{-}^{\prime \prime}\right)\right]}{\Omega^{2}\left(\Omega^{2}+\gamma_{*}^{-4} \delta_{\lambda}^{2}-2 \kappa_{\lambda} \gamma_{*}^{-2} \delta_{\lambda} \Omega\right)}\right\rangle \delta\left(\omega-\omega_{\mathrm{B}}^{\prime}\right),
\end{aligned}
$$




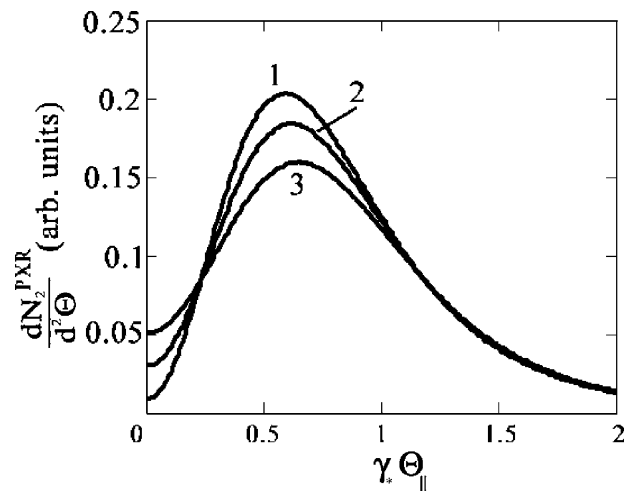

Fig. 3. Influence of the beam multiple scattering on the forward PXR angular distribution. Presented curves have been calculated for different values of the parameter $\gamma_{*} \Psi_{\mathrm{L}}: 1-\gamma_{*} \Psi_{\mathrm{L}}=0.1$; $2-\gamma_{*} \Psi_{\mathrm{L}}=0.2 ; 3-\gamma_{*} \Psi_{\mathrm{L}}=0.3$.

analogous to (13), follows from (11) within the frame of the approximation (12). The argument of $\delta$-function in (21) is determined with an accuracy of $\gamma^{-1}$.

Formula (21) shows that PXR spectral width depends strongly on a photon collimator size. Since $\omega_{\mathrm{B}}^{\prime}$ does not depend on the observation angle $\Theta_{\perp}$ (see formula (10)), the collimator in a slit form with the finite angular size $\Delta \Theta_{\|}$is of interest from the view-point of the separation of weak PXR signal with narrow spectrum on TR background with wide spectrum. Assuming the conditions (17) and (20) to be valid one can perform the integration in (21) under the additional conditions $\kappa_{\lambda} \ll 1$ and $L / \cos (\varphi / 2) \gg 1 / \omega \chi_{0}^{\prime \prime}$. The result of integration has the form

$$
\begin{aligned}
\frac{\mathrm{d} N_{\lambda}^{\mathrm{PXR}}}{\mathrm{d} \omega}= & \frac{e^{2} \omega_{0}^{2}}{2 g^{2}} \frac{\delta_{\lambda}^{2}}{\gamma_{*}} \frac{\tan (\varphi / 2)}{\omega_{\mathrm{B}} \chi_{0}^{\prime \prime}} F_{\lambda}(\xi) \eta \\
& \times\left[\gamma_{*}\left(\Theta_{\|}+\frac{1}{2} \Delta \Theta_{\|}\right)-\xi\right] \eta \\
& \times\left[\xi-\gamma_{*}\left(\Theta_{\|}-\frac{1}{2} \Delta \Theta_{\|}\right)\right] \\
F_{1}(\xi)= & \frac{1}{\sqrt{1+\xi^{2}}} \\
& -\frac{\sqrt{2}}{\delta_{1}} \sqrt{\sqrt{\left(1+\xi^{2}\right)^{2}+\delta_{1}^{2}}-1-\xi^{2}},
\end{aligned}
$$

$$
\begin{aligned}
F_{2}(\xi)=\xi^{2} & {\left[\frac{1}{\left(1+\xi^{2}\right)^{\frac{3}{2}}}\right.} \\
& \left.-\frac{\sqrt{2}}{\delta_{2}} \frac{\sqrt{\sqrt{\left(1+\xi^{2}\right)^{2}+\delta_{2}^{2}}-1-\xi^{2}}}{\sqrt{\left(1+\xi^{2}\right)^{2}+\delta_{2}^{2}}}\right]
\end{aligned}
$$

$\xi=\frac{\omega_{\mathrm{B}}^{\prime \prime}-\omega}{\omega_{0} \cot (\varphi / 2)}$,

$\omega_{\mathrm{B}}^{\prime \prime}=\omega_{\mathrm{B}}\left(1-\Theta_{\|}^{\prime} \cot (\varphi / 2)\right)$,

where the function $\eta(x)=1$ if $x>0$ and $\eta(x)=0$ if $x<0$.

There are two limitations on the forward PXR spectral width $\Delta \omega$ in accordance with (22). One of them is determined by the characteristics scale $\Delta \xi \sim$ 1 of the functions $F_{\lambda}(\xi)$, or $\Delta \omega \sim \omega_{0} \cot (\varphi / 2)$. The second one $\Delta \omega \approx \omega_{\mathrm{B}} \Delta \Theta_{\|} \cot (\varphi / 2)$ is determined by the collimator size $\Delta \Theta_{\|}$. Obviously

$\Delta \omega \approx\left\{\begin{array}{ll}\omega_{0} \cot (\varphi / 2) & \text { if } \Delta \Theta_{\|} \gg \gamma_{*}^{-1} \\ \omega_{\mathrm{B}} \Delta \Theta_{\|} \cot (\varphi / 2) & \text { if } \Delta \Theta_{\|} \ll \gamma_{*}^{-1}\end{array}\right.$.

Within the frame of approximation (12) the width $\Delta \omega \rightarrow 0$ when $\Delta \Theta_{\|} \rightarrow 0$ in accordance with (23). More general result (11) predicts the finite value of "natural" PXR spectral width even through the photon collimator angular size $\Delta \Theta_{\|} \rightarrow$ 0 . Using (11) one can obtain in the case of thick enough target $L / \cos (\varphi / 2) \gg 1 / \omega_{\mathrm{B}} \chi_{0}^{\prime \prime}$ the following estimation:

$\Delta \omega_{\min } \approx \omega_{\mathrm{B}} \frac{\chi_{0}^{\prime \prime}}{2 \sin ^{2}(\varphi / 2)}$,

so that the last formula in (23) is valid under condition $\Delta \Theta_{\|} \gg \chi_{0}^{\prime \prime} / \sin \varphi$ only.

The most interesting result, following from (22d), consists in the character of the average forward PXR photon energy $\langle\omega\rangle=\omega_{\mathrm{B}}^{\prime \prime}\left(\Theta_{\|}^{\prime}\right)$. In accordance with (22d) $\langle\omega\rangle$ decreases when increasing of $\Theta_{\|}^{\prime}$ in contrast with that for PXR photons emitted to Bragg scattering direction. Such a difference is caused by the fixed value of 


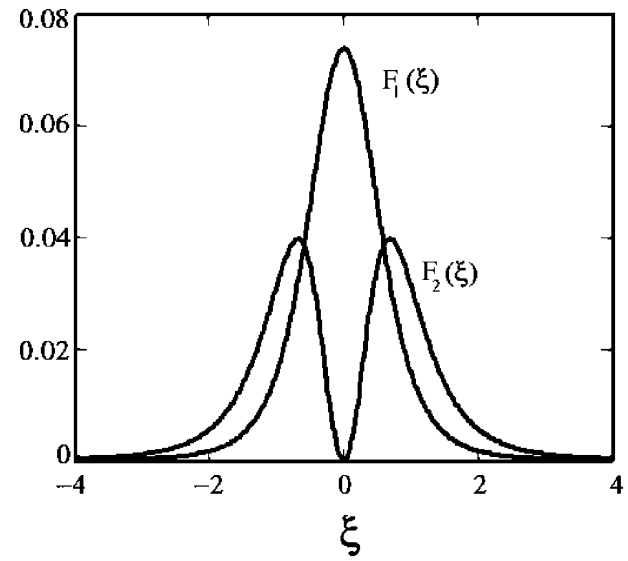

Fig. 4. The spectral distribution of non-collimated PXR for different polarizations. The curves have been calculated for the fixed parameters $\delta_{1}=0.88$ and $\delta_{2}=0.8$.

emission angle $\varphi$ between the axis of an electron beam and the axis of photon detector in the experiment devoted to the observation of PXR to Bragg scattering direction. Indeed, in the case of the forward PXR observation the equality $\omega=$ $\omega_{\mathrm{B}}^{\prime \prime}\left(\Theta_{\|}^{\prime}\right)$ follows from the condition $\Delta=0(\Delta=$ $\frac{1}{2} g^{2}+\omega \mathbf{n}_{\|} \mathbf{g}=\frac{1}{2} g^{2}-\omega g \sin \left(\frac{\varphi}{2}+\Theta_{\|}^{\prime}\right)$ is the Bragg resonance defect, defined in (5)) with the understanding that $\Theta=0$. As this takes place, $\omega \approx$ $\omega_{\mathrm{B}}\left(1-\Theta_{\|}^{\prime} \cot (\varphi / 2)\right)$. On the other hand the equation $\Delta=0$ has the form $\Delta=\frac{1}{2} g^{2}-\omega g \sin \left(\frac{\varphi}{2}-\right.$ $\left.\Theta_{\|}^{\prime}\right)=0$ in the case of PXR to Bragg scattering direction as is easy to show by Fig. 1. As might be expected, $\omega \approx \omega_{\mathrm{B}}\left(1+\Theta_{\|}^{\prime} \cot (\varphi / 2)\right)$ under these conditions.

In accordance with (22) the form of PXR spectral distribution depends strongly on the photon collimator angular size $\Delta \Theta_{\|}$and polarization index $\lambda$. In the case $\gamma_{*} \Delta \Theta_{\|} \ll 1$ the spectral variable $\xi(\omega)$ in (22d) is approximately equal to $\gamma_{*} \Theta_{\|}$. As this takes place the functions $F_{1}\left(\Theta_{\|}\right)$and $F_{2}\left(\Theta_{\|}\right)$determine the amplitudes of narrow PXR peaks with corresponding polarization. In the opposite case $\gamma_{*} \Delta \Theta_{\|} \gg 1$ the functions $F_{\lambda}(\xi)$ describe the natural PXR spectrum. These functions are illustrated by the curves presented in Fig. 4.

Let us consider now TR properties. Using (9b), one can obtain the following expression for TR spectral-angular distribution:

$$
\begin{aligned}
& \omega \frac{\mathrm{d} N_{\lambda}^{\mathrm{TR}}}{\mathrm{d} \omega \mathrm{d}^{2} \Theta} \\
&=\frac{e^{2}}{\pi^{2}}\left\langle\left(\mathbf{e}_{\lambda 0} \mathbf{v}\right)^{2}\left(\frac{1}{\Omega_{0}}-\frac{1}{\Omega}\right)^{2}\right. \\
& \times\left[1+\exp \left(-\frac{\omega L \chi_{0}^{\prime \prime}}{\cos (\varphi / 2)}\right)\right. \\
& \times\left(1-\frac{\alpha_{\lambda}^{2}}{\tau^{2}+\alpha_{\lambda}^{2}} \sin ^{2} Q_{1}\right)-2 \exp \left(-\frac{\omega L \chi_{0}^{\prime \prime}}{\cos (\varphi / 2)}\right) \\
&\left.\left.\quad \times\left(\cos Q_{1} \cos Q_{2}+\frac{\tau}{\sqrt{\tau^{2}+\alpha_{\lambda}^{2}}} \sin Q_{1} \sin Q_{2}\right)\right]\right\rangle \\
& Q_{1}=\frac{\omega_{\mathbf{g}}^{2} L}{2 \omega \cos (\varphi / 2)} \sqrt{\tau^{2}+\alpha_{\lambda}^{2}}, \\
& Q_{2}=\frac{\omega_{\mathbf{g}}^{2} L}{2 \omega \cos (\varphi / 2)}\left(\tau+\frac{\omega^{2}}{\omega_{\mathbf{g}}^{2}} \Omega\right) .
\end{aligned}
$$

It was suggested that the Bragg frequency $\omega_{\mathrm{B}}$ was far from the frequency range where the Borrmann effect can be realized (so that $\kappa_{\lambda} \ll 1$ ) when deriving (25) from (9b).

The expression (25) differs essentially from that describing TR from an amorphous dielectric plate, but such a difference takes place in the narrow vicinity of the Bragg frequency $\omega_{\mathrm{B}}$ only. Under condition $|\tau| \gg 1$ the distribution (25) arranges itself into the typical form

$$
\begin{aligned}
\omega \frac{\mathrm{d} N_{\lambda}^{\mathrm{TR}}}{\mathrm{d} \omega \mathrm{d}^{2} \Theta}=\frac{e^{2}}{\pi^{2}} & \left\langle\left(\mathbf{e}_{\lambda 0} \mathbf{v}\right)^{2}\left(\frac{1}{\Omega_{0}}-\frac{1}{\Omega}\right)^{2}\right. \\
\times & {\left[1+\exp \left(-\frac{\omega L \chi_{0}^{\prime \prime}}{\cos (\varphi / 2)}\right)\right.} \\
& -2 \exp \left(-\frac{\omega L \chi_{0}^{\prime \prime}}{2 \cos (\varphi / 2)}\right) \\
& \left.\left.\times \cos \left(\frac{\omega L}{2 \cos (\varphi / 2)} \Omega\right)\right]\right\rangle .
\end{aligned}
$$

TR contribution in the vicinity of Bragg frequency and observation angle $\Theta_{\|} \approx(1 / \sqrt{3}) \gamma_{*}^{-1} \gg$ $\gamma^{-1}$, where the maximum of PXR peak is realized, is of most interest for the purpose of PXR reflex observation. In the case of the target with small thickness $L<\cos (\varphi / 2) / \omega \chi_{0}^{\prime \prime}$ an interference between two TR waves emitted from in and outsurfaces of the target allows to suppress basically 


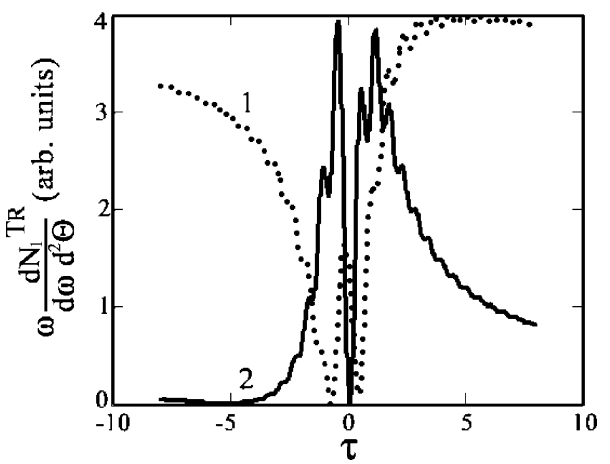

Fig. 5. The spectral-angular distribution of TR from thin nonabsorbing target. The curves have been calculated for fixed parameter $\left(\omega^{2} / \omega_{\mathbf{g}}^{2}\right) \Omega=2$ and different values of the parameter $\omega_{\mathrm{g}}^{2} L / 2 \omega \cos (\varphi / 2): 1-\omega_{\mathrm{g}}^{2} L / 2 \omega \cos (\varphi / 2)=5 ; 2-\omega_{\mathrm{g}}^{2} L / 2 \omega \cos (\varphi /$ 2) $=6$.

the discussed local TR contribution. But TR crosssection (25) oscillates strongly as a function of $\Theta$ and $\omega$ (see Fig. 5) in the vicinity of $\omega_{\mathrm{B}}$. Since the scale of such oscillations is comparable to that of the forward PXR peak, it is very difficult to both realize the condition of TR suppression and control this condition in an experiment. The discussed oscillations are realized on the background, determined by (26). This background can be reduced on the condition

$\frac{\omega_{\mathrm{B}} L}{2 \cos (\varphi / 2)} \Omega=2 \pi n, \quad n=1,2, \ldots$,

as it follows from (26). But on the other hand the strong narrow TR peak appears in the vicinity of $\omega_{\mathrm{B}}$ on condition under consideration due to dynamical diffraction effects [17]. Such a peak, analyzed in [17], contributes to the frequency and angular range, coinciding with that of PXR. Moreover, this peak dominates in the angular range, $\Theta \sim \gamma^{-1} \ll \gamma_{*}^{-1}$.

In the case of large enough thickness of crystalline target $L>2 \cos (\varphi / 2) / \omega_{\mathrm{B}} \chi_{0}^{\prime \prime}$, when a photoabsorption comes in to particular prominence PXR yield is saturated and an influence of TR resonance properties is not essential. Under such conditions the most important for the experiment question consist in the calculation of the dependence of collimated PXR and TR spectra on the observation and orientation angles with account of an interference between these emission mecha- nisms. Interference effect is described by the formula

$$
\begin{aligned}
\omega \frac{\mathrm{d} N_{\lambda}^{\mathrm{INT}}}{\mathrm{d} \omega \mathrm{d}^{2} \Theta}=- & \frac{e^{2} \alpha_{\lambda}^{2}}{\pi^{2}}\left\langle\frac{\left(\mathbf{e}_{\lambda 0} \mathbf{v}\right)^{2}}{\Omega}\left(\frac{1}{\Omega_{0}}-\frac{1}{\Omega}\right)\right. \\
& \times \frac{\tau-\sqrt{\tau^{2}+\alpha_{\lambda}^{2}}+\frac{\omega^{2}}{\omega_{\mathrm{g}}^{2}} \Omega}{\left(\tau-\sqrt{\tau^{2}+\alpha_{\lambda}^{2}}+\frac{\omega^{2}}{\omega_{\mathrm{g}}^{2}} \Omega\right)^{2}+\left(\frac{\omega^{2}}{\omega_{\mathrm{g}}^{2}} \chi_{0}^{\prime \prime}\right)^{2}} \\
& \times \frac{1}{\sqrt{\tau^{2}+\alpha_{\lambda}^{2}}}\left[1-\exp \left(-\frac{\omega L \chi_{0}^{\prime \prime}}{2 \cos (\varphi / 2)}\right)\right. \\
& \times \cos \left(Q_{2}-Q_{1}\right)-\exp \left(-\frac{\omega L \chi_{0}^{\prime \prime}}{2 \cos (\varphi / 2)}\right) \\
& \times\left(\cos Q_{1} \cos Q_{2}+\frac{\tau}{\sqrt{\tau^{2}+\alpha_{\lambda}^{2}}} \sin Q_{2} \sin Q_{1}\right) \\
& +\exp \left(-\frac{\omega L \chi_{0}^{\prime \prime}}{\cos (\varphi / 2)}\right) \\
& \left.\left.\times\left(\cos ^{2} Q_{1}+\frac{\tau}{\sqrt{\tau^{2}+\alpha_{\lambda}^{2}}} \sin ^{2} Q_{1}\right)\right]\right\rangle,
\end{aligned}
$$

following from (9) under condition $\kappa_{\lambda} \ll 1$.

Formulae (11), (25) and (28) allow to elucidate the most appropriate conditions for the forward PXR observation.

\section{Discussion of experimental conditions}

In accordance with obtained theoretical results the forward PXR characteristics depend on many parameters. First of all it should be pointed to the limitation on the emitting particle energy $\epsilon \gg \gamma_{*} m$ (17) following from (16). Since the typical for used in experiments crystals value of $\gamma_{*} m$ is of the order of hundreds $\mathrm{MeV}$ an electron beam with particle's energy of the order of $1 \mathrm{GeV}$ is required for experimental investigations of the forward PXR. In the following, we will assume the condition (17) to be valid.

The forward PXR can best be demonstrated from an experiment in which the emission properties are controlled by one parameter only; in this case only PXR properties must be changed when changing of such a parameter whereas TR contribution remains fixed. Such experimental conditions can be realized due to PXR and TR 
characteristics described above in the previous section.

In accordance with (13), (16) and (18) PXR angular distribution does not depend on the orientation angles $\Theta_{\|}^{\prime}$ and $\Theta_{\perp}^{\prime}$. On the other hand PXR spectral distribution depends strongly on the orientation angle $\Theta_{\|}^{\prime}$ and does not depend on the angle $\Theta_{\perp}^{\prime}$ as it follows from (21) and (22). As for TR spectral and angular properties they become very simple with the understanding that $L \gg$ $2 \cos (\varphi / 2) / \omega_{\mathrm{B}} \chi_{0}^{\prime \prime}$, when TR yield coincides with that emitted from out-surface of the target (see formula (25) in the corresponding limit). On condition under consideration TR angular distribution does not depend on the orientation angles $\Theta_{\|}^{\prime}$ and $\Theta_{\perp}^{\prime}$ and furthermore TR spectral distribution is approximately constant in the vicinity of the Bragg frequency $\omega_{\mathrm{B}}$ where PXR peak is realized.

Starting from the described emission properties we can say with reasonable confidence that the orientation angle $\Theta_{\|}^{\prime}$ is the best parameter for emission characteristics operating with the goal of a separation of PXR contribution on TR background. Indeed, only the position of PXR spectral peak $\omega=\omega_{\mathrm{B}}^{\prime}\left(\Theta_{\|}^{\prime}\right)$ can be changed when changing of $\Theta_{\|}^{\prime}$ in the experiment with fixed other parameters, such as average values of observation angles $\Theta_{\|}=\left\langle\Theta_{\|}\right\rangle, \Theta_{\perp}=\left\langle\Theta_{\perp}\right\rangle=0$, orientation angle $\Theta_{\perp}^{\prime}=$ 0 and collimator size $\Delta \Theta_{\|} \Delta \Theta_{\perp}=$ const. Parameters $\left\langle\Theta_{\|}\right\rangle, \Delta \Theta_{\perp}$ and $\Delta \Theta_{\|}$must be calculated theoretically in order to obtain the best ratio signal/ background in the experiment.

Since PXR spectral width depends strongly on the collimator size $\Delta \Theta_{\|}$we will use in calculations the general formula (11) taking into account "natural" spectral width (24). To obtain a convenient formula for PXR contribution estimations we will assume that the photon collimator size $\Delta \Theta_{\|}$is small relative to the observation angle $\left\langle\Theta_{\|}\right\rangle$ (in accordance with (16) PXR angular maximum is achieved in the range of large enough angles $\left.\Theta_{\|} \sim \gamma_{*}^{-1}\right)$. This condition ensured that the integration over $\mathrm{d} \Theta_{\|}$in (11) can be performed on the assumption that $\Theta_{\|} \approx\left\langle\Theta_{\|}\right\rangle$in the function $\Omega\left(\Theta_{\|}, \Theta_{\perp}\right)$ and the dependence of PXR spectralangular distribution on $\Theta_{\|}$is concentrated in the fast variable $\tau\left(\omega, \Theta_{\|}^{\prime}, \Theta_{\|}\right)$. Let us assume that the target thickness $L$ is small enough so that the multiple scattering can be neglected (see the condition (20)). On the other hand such a thickness must exceed an absorption length $(L \gg$ $\left.2 \cos (\varphi / 2) / \omega_{\mathrm{B}} \chi_{0}^{\prime \prime}\left(\omega_{\mathrm{B}}\right)\right)$ so that PXR yield is saturated. Both these conditions can be represented as

$\frac{2 \cos (\varphi / 2)}{\omega_{\mathrm{B}} \chi_{0}^{\prime \prime}\left(\omega_{\mathrm{B}}\right)} \ll L \ll \frac{\gamma^{2}}{\gamma_{*}^{2}} \frac{e^{2}}{4 \pi} L_{\mathrm{R}}$.

Evidently, the field of application of (29) increases when increasing of an emission particle energy.

In the case under study the spectral distribution of PXR contribution to total emission yield is presented by

$$
\begin{aligned}
\frac{\mathrm{d} N^{\mathrm{PXR}}}{\mathrm{d} \omega}= & \frac{e^{2}}{2 \pi^{2}} \frac{\omega_{0} \tan (\varphi / 2)}{g^{2}} \sum_{\lambda=1}^{2} \delta_{\lambda} \int_{-\frac{1}{2} \gamma_{*} \Delta \Theta_{\perp}}^{\frac{1}{2} \gamma_{*} \Delta \Theta_{\perp}} \mathrm{d} y \frac{\gamma_{*}^{2} \Theta_{\lambda}^{2}}{\Omega^{2}} \\
& \times \int_{\tau_{\lambda-}}^{\tau_{\lambda+}} \frac{\mathrm{d} x}{x^{2}+1} \\
& \times \frac{1}{\left(x-\sqrt{x^{2}+1}+\Omega / \delta_{\lambda}\right)^{2}+\left(\gamma_{*}^{2} \chi_{0}^{\prime \prime} / \delta_{\lambda}\right)^{2}}, \\
\tau_{\lambda \pm}=(1 & -\cos \varphi) \frac{\gamma_{*}^{2}}{\delta_{\lambda}}\left(1-\frac{\omega}{\omega_{\mathrm{B}}^{\prime}}\right) \pm \sin \varphi \frac{\gamma_{*}^{2}}{2 \delta_{\lambda}} \Delta \Theta_{\|},
\end{aligned}
$$

where $\Omega=1+\gamma_{*}^{2}\left\langle\Theta_{\|}\right\rangle^{2}+\gamma_{*}^{2} / \gamma^{2}+y^{2}, \quad \gamma_{*}^{2} \Theta_{1}^{2}=y^{2}$, $\gamma_{*}^{2} \Theta_{2}^{2}=\gamma_{*}^{2}\left\langle\Theta_{\|}\right\rangle^{2}$, the quantity $\omega_{\mathrm{B}}^{\prime}$ is defined by the corresponding formula in (10) where the angle $\left\langle\Theta_{\|}\right\rangle$is used instead of $\Theta_{\|}$.

TR background is described by the formula

$$
\begin{aligned}
\frac{\mathrm{d} N^{\mathrm{TR}}}{\mathrm{d} \omega}= & \frac{2 e^{2}}{\pi^{2}} \frac{\omega_{0} \tan (\varphi / 2)}{g^{2}} \\
& \times \sin \varphi \gamma_{*}^{2} \Delta \Theta_{\|}\left[\frac{1}{\sqrt{\gamma_{*}^{2}\left\langle\Theta_{\|}\right\rangle^{2}+\gamma_{*}^{2} / \gamma^{2}}}\right. \\
& \times\left(1+\frac{\gamma_{*}^{2}\left\langle\Theta_{\|}\right\rangle^{2}}{\gamma_{*}^{2}\left\langle\Theta_{\|}\right\rangle^{2}+\gamma_{*}^{2} / \gamma^{2}}+4 \gamma_{*}^{2} / \gamma^{2}\right) \\
& \times \arctan \left(\frac{\gamma_{*} \Delta \Theta_{\perp}}{2 \sqrt{\gamma_{*}^{2}\left\langle\Theta_{\|}\right\rangle^{2}+\gamma_{*}^{2} / \gamma^{2}}}\right) \\
& -\frac{1}{\sqrt{1+\gamma_{*}^{2}\left\langle\Theta_{\|}\right\rangle^{2}+\gamma_{*}^{2} / \gamma^{2}}}
\end{aligned}
$$




$$
\begin{aligned}
& \times\left(2+\frac{1+\gamma_{*}^{2} / \gamma^{2}}{1+\gamma_{*}^{2}\left\langle\Theta_{\|}\right\rangle^{2}+\gamma_{*}^{2} / \gamma^{2}}+4 \gamma_{*}^{2} / \gamma^{2}\right) \\
& \times \arctan \left(\frac{\gamma_{*} \Delta \Theta_{\perp}}{2 \sqrt{1+\gamma_{*}^{2}\left\langle\Theta_{\|}\right\rangle^{2}+\gamma_{*}^{2} / \gamma^{2}}}\right) \\
& -\frac{1}{2} \frac{\gamma_{*}^{2} / \gamma^{2}}{\gamma_{*}^{2}\left\langle\Theta_{\|}\right\rangle^{2}+\gamma_{*}^{2} / \gamma^{2}} \frac{\gamma_{*} \Delta \Theta_{\perp}}{\gamma_{*}^{2}\left\langle\Theta_{\|}\right\rangle^{2}+\gamma_{*}^{2} / \gamma^{2}+\frac{1}{4} \gamma_{*}^{2}\left(\Delta \Theta_{\perp}\right)^{2}} \\
& -\frac{1}{2} \frac{1+\gamma_{*}^{2} / \gamma^{2}}{1+\gamma_{*}^{2}\left\langle\Theta_{\|}\right\rangle^{2}+\gamma_{*}^{2} / \gamma^{2}} \\
& \left.\times \frac{\gamma_{*} \Delta \Theta_{\perp}}{1+\gamma_{*}^{2}\left\langle\Theta_{\|}\right\rangle^{2}+\gamma_{*}^{2} / \gamma^{2}+\frac{1}{4} \gamma_{*}^{2}\left(\Delta \Theta_{\perp}\right)^{2}}\right]
\end{aligned}
$$

following from (25) at the same conditions.

It should be noted that the result (31) describes TR spectrum in the vicinity of the Bragg frequency only, but this limitation is not very essential because TR spectrum is approximately constant within the range $\omega \leqslant \omega_{\mathrm{B}}$ on condition (17) under consideration.

To describe correctly experimental data it is necessary to take into account an interference between TR and PXR. The corresponding formula follows from (28) in the form

$$
\begin{aligned}
\frac{\mathrm{d} N^{\mathrm{INT}}}{\mathrm{d} \omega}= & -\frac{2 e^{2}}{\pi^{2}} \frac{\omega_{0} \tan (\varphi / 2)}{g^{2}} \sum_{\lambda=1}^{2} \delta_{\lambda} \int_{-\frac{1}{2} \gamma_{*} \Delta \Theta_{\perp}}^{\frac{1}{2} \gamma_{*} \Delta \Theta_{\perp}} \mathrm{d} y \frac{\gamma_{*}^{2} \Theta_{\lambda}^{2}}{\Omega} \\
& \times\left(\frac{1}{\Omega_{0}}-\frac{1}{\Omega}\right) \int_{\tau_{\lambda-}}^{\tau_{\lambda+}} \frac{\mathrm{d} x}{\sqrt{x^{2}+1}} \\
& \times \frac{x-\sqrt{x^{2}+1}+\Omega / \delta_{\lambda}}{\left(x-\sqrt{x^{2}+1}+\Omega / \delta_{\lambda}\right)^{2}+\left(\gamma_{*}^{2} \chi_{0}^{\prime \prime} / \delta_{\lambda}\right)^{2}},
\end{aligned}
$$

where $\Omega_{0}=\gamma_{*}^{2}\left\langle\Theta_{\|}\right\rangle^{2}+\gamma_{*}^{2} / \gamma^{2}+y^{2}$.

Let us use the formulae (30)-(32) to describe expected results of experimental study of the forward PXR from different crystals. Keeping in mind an investigation of an influence of the target atomic number $Z$ on emission characteristics we will consider PXR from heave (W) and light (Si) crystalline targets.

In accordance with the formulae (30)-(32) the experimental parameters $\omega_{\mathrm{B}}, \Delta \Theta_{\perp}, \Delta \Theta_{\|},\left\langle\Theta_{\|}\right\rangle$appear in these formulae in the form of combinations $\gamma_{*} / \gamma=\omega_{\mathrm{B}} / \gamma \omega_{0}, \gamma_{*} \Delta \Theta_{\perp}, \sin \varphi \gamma_{*}^{2} \Delta \Theta_{\|}, \gamma_{*}\left\langle\Theta_{\|}\right\rangle$. Choos-

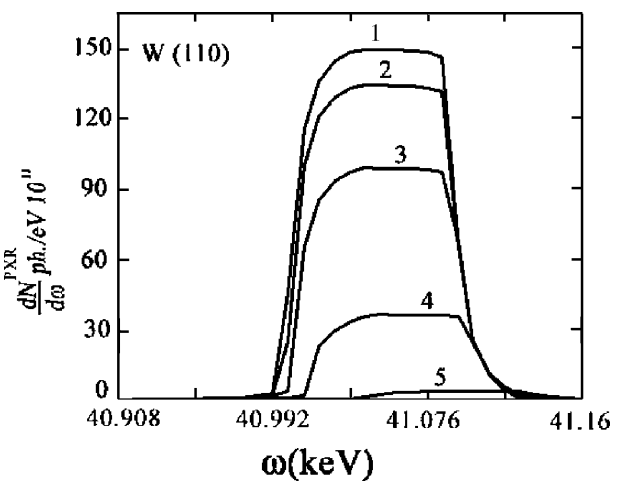

Fig. 6. The forward PXR spectrum from $\mathrm{W}$ crystal versus the energy of an emitting particle. The presented spectral distributions have been calculated for fixed parameters: $\omega_{\mathrm{B}}=42 \mathrm{keV}$ $(\varphi=0.134 \mathrm{rad}) ; \gamma_{*}=500 ; \gamma_{*} \Delta \Theta_{\perp}=4 ; \gamma_{*}\left\langle\Theta_{\|}\right\rangle=0.8 ; \gamma_{*} \Theta_{\|}^{\prime}=0$ and $\sin \varphi \gamma_{*}^{2} \Delta \Theta_{\|}=5$. The values of electron Lorentz factor are: $1-\gamma \gg \gamma_{*} ; 2-\gamma=2 \times 10^{3} ; 3-\gamma=10^{3} ; 4-\gamma=500 ; 5-\gamma=250$.

ing the Bragg energy $\omega_{\mathrm{B}}$ one should take into account that the particle energy $\epsilon=\gamma m$ must be greater than the critical energy $\gamma_{*} m$ (see (17)). To illustrate an importance of the condition (17) we present in Fig. 6 the spectrum of PXR photons emitted from $\mathrm{W}(110)$ for different values of the parameter $\gamma_{*} / \gamma$ and fixed others parameters. Presented curves show that PXR yield increases when increasing of $\gamma$ and saturates in the region $2 \gamma_{*} \leqslant \gamma$. On the other hand the ratio signal/background following from the total emission spectrum

$\frac{\mathrm{d} N}{\mathrm{~d} \omega}=\frac{\mathrm{d} N^{\text {PXR }}}{\mathrm{d} \omega}+\frac{\mathrm{d} N^{\mathrm{TR}}}{\mathrm{d} \omega}+\frac{\mathrm{d} N^{\mathrm{INT}}}{\mathrm{d} \omega}$

depends only slightly on the parameter $\gamma_{*} / \gamma$, as it is demonstrated by the curves presented in Fig. 7. Therefore the most appropriate value of the Bragg frequency $\omega_{\mathrm{B}}$ is determined by the condition

$\omega_{\mathrm{B}} \approx \frac{1}{2} \gamma \omega_{0}$.

Since PXR yield is proportional to an absorption length (see formula (22a)) which increases with increasing of the emitted photon energy $\omega \approx \omega_{\mathrm{B}}$ the value of $\omega_{\mathrm{B}}$ in (34) should be considered as maximum possible one for given electron energy $\gamma_{*} m$.

The curves, presented in Fig. 8, illustrate an influence of the photon collimator angular size $\Delta \Theta_{\perp}$ on the total emission spectrum (33). As 


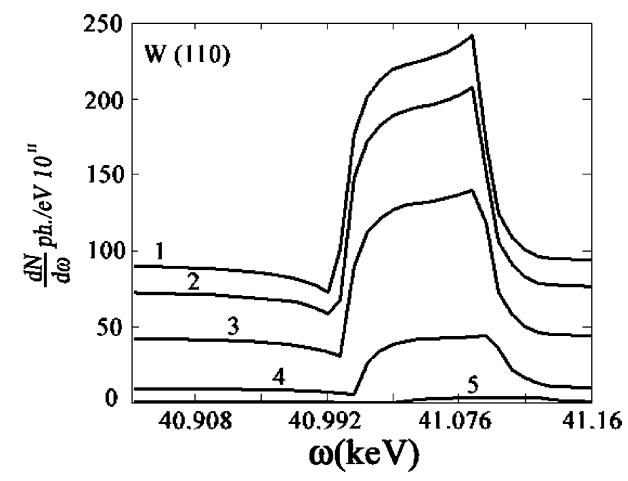

Fig. 7. The same, but for the total emission spectrum including PXR, TR and interference term.

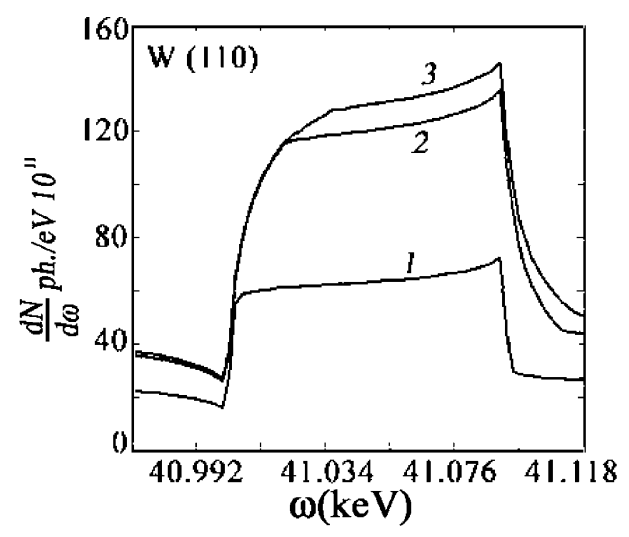

Fig. 8. The dependence of the total emission spectrum on the photon collimator angular size $\Delta \Theta_{\perp}$. Presented curves have been calculated for fixed parameters $\omega_{\mathrm{B}}=42 \mathrm{keV}(\varphi=0.134$ $\mathrm{rad}) ; \quad \gamma_{*} / \gamma=0.5 ; \quad \gamma_{*}=500 ; \quad \gamma_{*}\left\langle\Theta_{\|}\right\rangle=0.8 ; \quad \sin \varphi \gamma_{*}^{2} \Delta \Theta_{\|}=5$ $\gamma_{*} \Theta_{\|}^{\prime}=0$ and different values of the parameter $\gamma_{*} \Delta \Theta_{\perp}: 1-$ $\gamma_{*} \Delta \Theta_{\perp}=1 ; 2-\gamma_{*} \Delta \Theta_{\perp}=2.8 ; 3-\gamma_{*} \Delta \Theta_{\perp}=4$.

would be expected, the yield saturates when $\Delta \Theta_{\perp}$ exceeds the emission characteristic angular scale $\gamma_{*}^{-1}$. In accordance with presented curves the position of PXR reflex, its spectral width and the ratio signal/background are little affected with changing of $\Delta \Theta_{\perp}$.

The next question of specific interest for the forward PXR experimental verification consists in correct choosing the observation angle $\left\langle\Theta_{\|}\right\rangle$, which is the main factor determining the ratio signal/ background. The curves presented in Fig. 9 demonstrate an essential growth of such a ratio when increasing of the angle $\left\langle\Theta_{\|}\right\rangle$. On the other hand

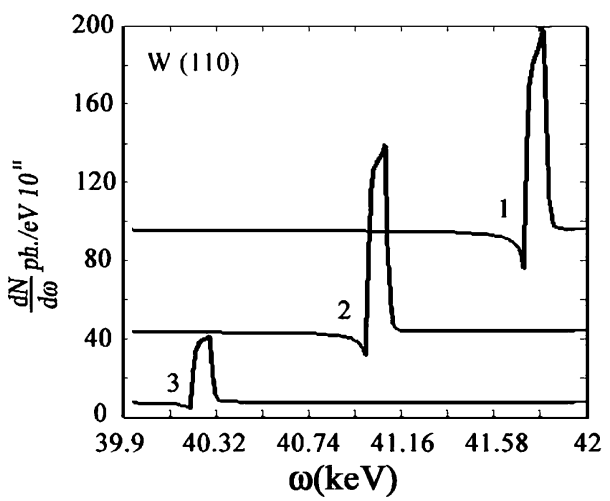

Fig. 9. The dependence of the total emission spectrum on the average observation angle $\left\langle\Theta_{\|}\right\rangle$. The curves presented in this figure have been calculated for fixed parameters $\omega_{\mathrm{B}}=$ $42 \mathrm{keV}(\varphi=0.134 \mathrm{rad}) ; \gamma_{*}=500 ; \gamma_{*} / \gamma=0.5 ; \gamma_{*} \Delta \Theta_{\perp}=4$; $\sin \varphi \gamma_{*}^{2} \Delta \Theta_{\|}=5 ; \gamma_{*} \Theta_{\|}^{\prime}=0$ and different values of the parameter $\gamma_{*}\left\langle\Theta_{\|}\right\rangle: 1-\gamma_{*}\left\langle\Theta_{\|}\right\rangle=0.2 ; 2-\gamma_{*}\left\langle\Theta_{\|}\right\rangle=0.8 ; 3-\gamma_{*}\left\langle\Theta_{\|}\right\rangle=1.5$.

this growth is accompanied by decreasing of a total emission yield resulting on difficulties in a measuring process due to the external background. It should be noted that the influence of an interference between PXR and TR decreases with increasing of $\left\langle\Theta_{\|}\right\rangle$in accordance with Fig. 9. Such behaviour of discussed spectra are explained by the difference between PXR and TR angular distributions. Indeed, TR angular distribution is more narrow than PXR one on condition $\gamma>\gamma_{*}$ under consideration. As this takes place TR contribution dominates in the region of relatively small observation angles $\left\langle\Theta_{\|}\right\rangle \sim \gamma^{-1}<\gamma_{*}^{-1}$. On the other hand PXR dominates in the range $\left\langle\Theta_{\|}\right\rangle \gamma_{*}^{-1}$ on the same condition. Therefore the relative contribution of PXR to total emission yield increases with increasing of $\left\langle\Theta_{\|}\right\rangle$, but the contribution of interference term (32), proportional to the product of TR and PXR amplitudes, decreases relative to PXR yield.

An influence of the photon collimator angular size $\Delta \Theta_{\|}$in the emission spectrum (33) is illustrated by the curves, presented in Fig. 10. It contrast with that for $\Delta \Theta_{\perp}$ the growth of $\Delta \Theta_{\|}$will cause the PXR spectral width to increase. The total emission yield increases, but the ratio signal/ background decreases when increasing of $\Delta \Theta_{\|}$, as it follows from Fig. 10. Therefore the value of $\Delta \Theta_{\|}$ must be chosen based on the conditions of real 


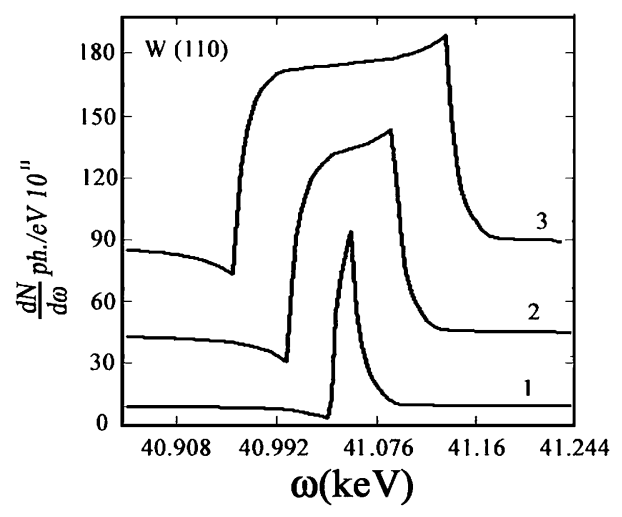

Fig. 10. The total emission spectrum versus the photon collimator angular size $\Delta \Theta_{\|}$. Presented curves have been calculated for fixed parameters $\omega_{\mathrm{B}}=42 \mathrm{keV}(\varphi=0.134 \mathrm{rad}) ; \gamma_{*}=500$; $\gamma_{*}\left\langle\Theta_{\|}\right\rangle=0.8 ; \gamma_{*} / \gamma=0.5 ; \gamma_{*} \Delta \Theta_{\perp}=4 ; \gamma_{*} \Theta_{\|}^{\prime}=0$ and different values of the parameter $\sin \varphi \gamma_{*}^{2} \Delta \Theta_{\|}: 1-\sin \varphi \gamma_{*}^{2} \Delta \Theta_{\|}=1 ; 2-$ $\sin \varphi \gamma_{*}^{2} \Delta \Theta_{\|}=5 ; 3-\sin \varphi \gamma_{*}^{2} \Delta \Theta_{\|}=10$.

experiment. It should be noted that the formula (30), describing PXR spectrum, has been obtained on the assumption that $\Delta \Theta_{\|} \ll\left\langle\Theta_{\|}\right\rangle \sim \gamma_{*}^{-1}$. One can see, that such a condition may be easy fulfilled because the coefficient $\sin \varphi \gamma_{*}^{2} \Delta \Theta_{\|}$, determining PXR spectral width, can be represented in the form $\left(g \cos (\varphi / 2) / \omega_{0}\right) \gamma_{*} \Delta \Theta_{\|}, g \cos (\varphi / 2) / \omega_{0} \gg 1$.

Let us consider now the main for our purposes dependence of the total emission spectrum (33) on the orientation angle $\Theta_{\|}^{\prime}$. This dependence, calculated for fixed parameters $\gamma_{*} \Delta \Theta_{\perp}, \gamma_{*}\left\langle\Theta_{\|}\right\rangle$and $\sin \varphi \gamma_{*}^{2} \Delta \Theta_{\|}$, is presented in Fig. 11. In accordance with the results of theoretical analysis, performed in the previous section, only the position of PXR reflex is changed with changing of the orientation angle $\Theta_{\|}^{\prime}$.

Obtained in this section numerical results describe the forward PXR from W crystalline target. The dependence of emission characteristics on the target atomic number $Z$ is still to be determined. To consider this question we will analyze an emission properties from $\operatorname{Si}(220)$ crystal.

For correct comparison of $\mathrm{W}$ and Si crystals as the forward PXR radiators we assume that Bragg frequencies for both cases are determined by the Eq. (34), so that the coefficient $\gamma_{*} / \gamma$ is the same for $\mathrm{W}$ and Si radiators. Since W and Si crystals vary on the plasma frequency $\omega_{0}$ and the reciprocal lattice vector $\mathbf{g}$ the Bragg frequencies and orien-

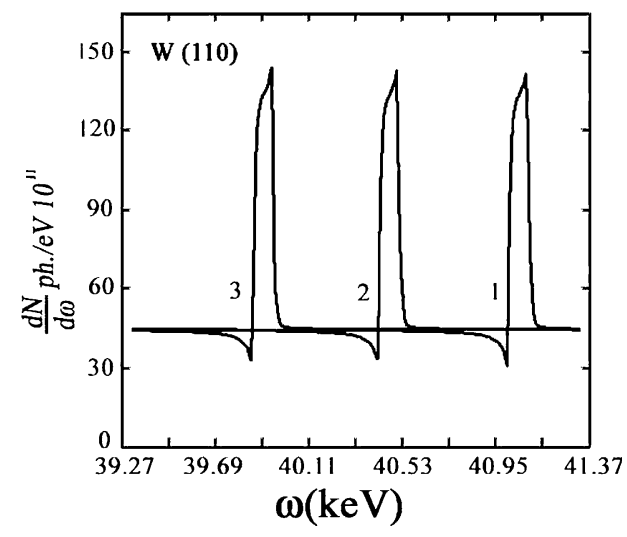

Fig. 11. The dependence of the forward PXR reflex on the orientation angle $\Theta_{\|}^{\prime}$. The positions of PXR peaks correspond the following values of the parameter $\gamma_{*} \Theta_{\|}^{\prime}: 1-\gamma_{*} \Theta_{\|}^{\prime}=0 ; 2-$ $\gamma_{*} \Theta_{\|}^{\prime}=0.5 ; 3-\gamma_{*} \Theta_{\|}^{\prime}=1$. The curves have been calculated for fixed parameters $\omega_{\mathrm{B}}=42 \mathrm{keV}(\varphi=0.134 \mathrm{rad}) ; \gamma_{*}=500$; $\gamma_{*}\left\langle\Theta_{\|}\right\rangle=0.8 ; \gamma_{*} / \gamma=0.5 ; \gamma_{*} \Delta \Theta_{\perp}=4 ; \sin \varphi \gamma_{*}^{2} \Delta \Theta_{\|}=5$.

tation angles between emitting electron impulse and reflecting crystallographic plane are different for these crystals due to the condition (34). It is reasonable to suggest that the parameters $\gamma_{*} \Delta \Theta_{\perp}$, $\gamma_{*}\left\langle\Theta_{\|}\right\rangle$and $\sin \varphi \gamma_{*}^{2} \Delta \Theta_{\|}$, determining the properties of distributions (30)-(32) apart from the parameter $\gamma_{*} / \gamma$, must be the same for $\mathrm{W}$ and $\mathrm{Si}$ for correct comparison of these radiators. Curves presented on Fig. 12 describe $\Delta \Theta_{\|}$-dependence of the total emission spectrum, calculated for $\operatorname{Si}(220)$ at the indicated conditions.

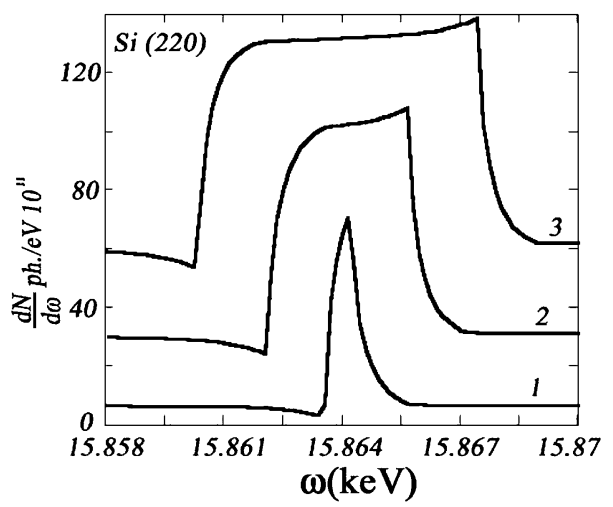

Fig. 12. The same as in Fig. 10, but for $\operatorname{Si}(220)$ target. The presented curves have been calculated for fixed parameters $\omega_{\mathrm{B}}=16 \mathrm{keV}(\varphi=0.413 \mathrm{rad}) ; \gamma_{*}=500 ; \gamma_{*}\left\langle\Theta_{\|}\right\rangle=0.9 ; \gamma_{*} \Theta_{\|}^{\prime}=0$; $\gamma_{*} / \gamma=0.5 ; \gamma_{*} \Delta \Theta_{\perp}=4$. The values of the parameter $\sin \varphi \gamma_{*}^{2} \Delta \Theta_{\|}$ are the same as in Fig. 10. 
Evidently, the discussed dependence is analogous to that, calculated for $\mathrm{W}$ radiator and presented in Fig. 10, but the spectral width for radiator is much more than that for $\mathrm{Si}$ one. The forward PXR spectral width $\Delta \omega \approx \omega_{\mathrm{B}} \Delta \Theta_{\|} \cot (\varphi / 2)$, determined by the Eq. (23) (this formula for $\Delta \omega$ is immediately follows from the equation $\omega=\omega_{\mathrm{B}}^{\prime}$, is defined by (10)), can be represented as

$$
\Delta \omega \approx \frac{\gamma \omega_{0}^{3}}{g^{2}} \sin \varphi \gamma_{*}^{2} \Delta \Theta_{\|}
$$

on condition (34) under consideration. In accordance with (35) $\Delta \omega \sim Z^{\frac{3}{2}}$ for fixed parameter $\sin \varphi \gamma_{*}^{2} \Delta \Theta_{\|}$, as expected in our analysis.

Two important for an experiment conclusions follow from (35) and Figs. 10 and 12. The total emission yield increases when increasing of the target atomic number $Z$ resulting in the growth of the ratio emission under study/external background. In addition to this in the experiment with Si target X-ray detector with very high energy resolution $\delta \omega$ is needed ( $\delta \omega \sim 1-10 \mathrm{eV}$ in accordance with Fig. 12) in contrast to the experiment with $\mathrm{W}$ target, when the value of $\delta \omega$ can be of the order of hundreds eV. Thus, W crystal is more convenient for the forward PXR observation than $\mathrm{Si}$ one.

Integrating (30) over the photon energies one can obtain the following formula:

$$
\begin{aligned}
N^{\mathrm{PXR}} & \approx \frac{e^{2}}{2} \frac{\omega_{0} \tan (\varphi / 2)}{g^{2}} \frac{\Delta \omega}{\gamma_{*}^{2} \chi_{0}^{\prime \prime}} G^{\mathrm{PXR}}, \\
G^{\mathrm{PXR}} & =\delta_{1}^{2}\left(\frac{1}{\Omega_{1}^{\frac{1}{2}}}-\frac{\sqrt{2}}{\sqrt{\sqrt{\Omega_{1}^{2}+\delta_{2}^{2}}+\Omega_{1}}}\right)+\delta_{2}^{2} \gamma_{*}^{2}\left\langle\Theta_{\|}\right\rangle^{2} \\
& \times\left(\frac{1}{\Omega_{1}^{\frac{3}{2}}}-\frac{\sqrt{2}}{\sqrt{\Omega_{1}^{2}+\delta_{2}^{2}} \sqrt{\sqrt{\Omega_{1}^{2}+\delta_{2}^{2}}+\Omega_{1}}}\right)
\end{aligned}
$$

where $\Omega_{1}=1+\gamma_{*}^{2}\left\langle\Theta_{\|}\right\rangle^{2}+\gamma_{*}^{2} / \gamma^{2}, \Delta \omega$ is determined by (35), this formula has been derived with the constraint $\gamma_{*} \Delta \Theta_{\perp} \gg 1$.

This formula allows not only to estimate the total number of emitted PXR photons $N^{\mathrm{PXR}}$, but to express the important ratio signal/background in terms of main parameters as well,

$$
\begin{aligned}
\frac{N^{\mathrm{PXR}}}{\Delta \omega} / \frac{N^{\mathrm{TR}}}{\mathrm{d} \omega}=\frac{\pi}{2} \frac{1}{\sin \varphi \gamma_{*}^{2} \Delta \Theta_{\|}} \frac{1}{\gamma_{*}^{2} \chi_{0}^{\prime \prime}}\left(G^{\mathrm{PXR}} / G^{\mathrm{TR}}\right), \\
G^{\mathrm{TR}}=\frac{1}{\sqrt{\Omega_{1}-1}}\left(1+\frac{\gamma_{*}^{2}\left\langle\Theta_{\|}\right\rangle^{2}}{\Omega_{1}-1}+4 \gamma_{*}^{2} / \gamma^{2}\right) \\
-\frac{1}{\sqrt{\Omega_{1}}}\left(2+\frac{1+\gamma_{*}^{2} / \gamma^{2}}{\Omega_{1}}+4 \gamma_{*}^{2} / \gamma^{2}\right),
\end{aligned}
$$

where the function $G^{\mathrm{TR}}$ has been obtained from (31) on condition $\gamma_{*} \Delta \Theta_{\perp} \gg 1$.

The last formula shows (in accordance with Figs. 10 and 12) that the photoabsorption and the photon collimator size $\Delta \Theta_{\|}$are the main parameters determining the ratio signal/background.

To test the validity of the developed theory we assume to perform the forward PXR experimental investigation at internal electron beam of Tomsk synchrotron $\left(E_{\mathrm{e}}^{\max }=500 \mathrm{MeV}, N_{\mathrm{e}} \leqslant 5 \times 10^{10}\right.$ electron per second) and external beam of Lebedev Physical Institute synchrotron with particle energies $300-1000 \mathrm{MeV}\left(N_{\mathrm{e}} \sim 10^{10}\right.$ electron per second), where we are planning to both search a dependence of PXR yield on the particle energy and measure an angular distribution of emitted photon flux.

In the previous experiment devoted to the forward PXR verification [15] an emission from $\mathrm{Si}$ crystal was measured for emitted photon energies 30 and $40 \mathrm{keV}$ and emitting electron energy $\epsilon=$ $500 \mathrm{MeV}$. Large energy of emitted photons $\left(\gamma_{*}>\right.$ $\gamma)$, small electron beam intensity and very narrow spectral width of the PXR peak in comparison with the energy resolution of used crystal-diffractometer $\left(\Delta \omega / \omega \sim 10^{-2}\right)$ prevented to separate the forward PXR contribution to total emission yield. As indicated in this paper, the most appropriate targets for the discussed emission observation are the crystals with large atomic numbers like tungsten. The width of the forward PXR spectral distribution for this crystal exceeds that for $\mathrm{Si}$ target under the comparable conditions by more than one order of magnitude in accordance with Figs. 10 and 12.

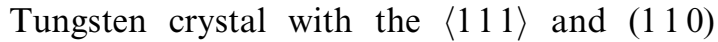
orientation will be used in the new experiment at Tomsk synchrotron. This crystal has been prepared by the use of the technology described in 
[20]. The crystal mosaicity does not exceed 0.5 mrad (FWHM). For the tungsten crystal with the thickness $0.3 \mathrm{~mm}$ fabricated by the use of this technology the effect of anomalous photoabsorption of $\mathrm{Fe} K_{\alpha}$ radiation has been observed in the experiment [21].

Experimental method used in the experiment [15] may appear as the most convenient for the forward PXR observation. As in the cited work two crystal-diffraction spectrometers based on pyrolytic graphite crystals and thin $\mathrm{NaI}(\mathrm{Tl})$ detectors $(t=1 \mathrm{~mm})$ will be used. These spectrometers ensure energy resolution $\Delta \omega / \omega \leqslant 1 \%$ and relatively high intensity of diffracted photon flux (for the first reflection order X-ray reflectivity is about $10-20 \%$ [22]). The contribution of background photons with energies different from the selected one do not exceed 2-5\%. High diffracted beam intensity is needed because of small intensity of the radiation under study and relatively low current of electron beam. Changing of the observation angle $\left\langle\Theta_{\|}\right\rangle$will be realized by the shift of goniometers with installed pyrolytic graphite crystals to the direction perpendicular to the axis of incident electron beam. The maximum value of this angle is limited by the size of the accelerator chamber output window $\left(\sim 6-8 \mathrm{mrad}, \approx 3-4 \gamma_{*}^{-1}\right)$.

For the reliable experimental confirmation of the forward PXR existence we simultaneously measure two X-ray yield orientation dependencies (OD) for different photon energies $\left(\omega<\gamma \omega_{\mathrm{p}} \approx 80\right.$ $\mathrm{keV}$ ) and OD for high energy photons. The measurement of hard radiation yield is necessary for both the control of experimental apparatus and the crystal target alignment by planar and axial channeling radiation yield measurement. To eliminate the masked contribution of a planar channeling radiation the measurement of OD for the forward PXR will be carried out for the $\langle 111\rangle$ axis intersection (for more details see [15]).

As can be seen from Fig. 11, the forward PXR photon energy $\omega\left(\Theta_{\|}^{\prime}\right)$ is smoothly changed with changing of the crystal plane orientation angle $\Theta_{\|}^{\prime}$. Therefore the sharp maximum must be manifested in OD for the total emission yield, measured by crystal-diffractometer tuned to the fixed photon energy $\omega_{\mathrm{d}}$, when the average PXR energy $\omega\left(\boldsymbol{\Theta}_{\|}^{\prime}\right)$ becomes equal to $\omega_{\mathrm{d}}$. Simultaneous measurement of OD for two different photon energies allows to clearly demonstrate the forward PXR peak existence. PXR peak with larger energy must be measured firstly with increase in the orientation angle $\Theta_{\|}^{\prime}$ in accordance with the formula for $\omega_{\mathrm{B}}^{\prime \prime}\left(\Theta_{\|}^{\prime}\right)$ in $(22 \mathrm{~d})$.

The measurements will be carried out for photon energies $\omega_{\mathrm{d}} \approx 40 \mathrm{keV}\left(\gamma \sim 2 \gamma_{*}\right)$ and $\omega_{\mathrm{d}} \approx 30$ $\operatorname{keV}\left(\gamma \sim 2.5 \gamma_{*}\right)$. By picking such values of the photon energy one should take into account both the condition (17) and the requirement of a small influence of emitting electron coherent azimuhal scattering on atomic strings (see, for example, [23]) on the measured OD. On the basis of the measurements of an emission yield with the average energy of $\omega=67 \mathrm{keV}$ from tungsten crystal with thickness of $1.7 \mathrm{~mm}$ and the electron energy $E_{\mathrm{e}}=500 \mathrm{MeV}$ we may assume that for this electron energy and photon frequency $\omega \sim 40 \mathrm{keV}$ $\left(\Theta_{\mathrm{B}} \sim 70 \mathrm{mrad}\right)$ the distortion of X-ray OD due to the mentioned mechanism will be negligible [24]. The most appropriate values of photon energies and observation angles will be adjusted by the exact calculation of the influence of azimutal multiple scattering on the forward PXR characteristics.

\section{Conclusions}

The performed analysis provides a physical ground for experimental searches of the new emission mechanisms, known as PXR along an emitting particle velocity, or the forward PXR.

One of the most difficult problems in the task of the forward PXR observation is a high level background from the side of TR. The ratio $N^{\mathrm{PXR}} / N^{\mathrm{TR}}$ can be increased by the collimation of a total emission at large observation angles, where PXR angular density can exceed TR one. But as this takes place PXR yield becomes too small to be observed experimentally. The possibility to solve this problem is shown in our work. The most important results of performed calculations consist in the following:

- The forward PXR yield may be sufficient for its experimental measurement exclusively with the 
understanding that the energy of emitting electrons exceeds the critical energy determined by the Eq. (17);

- The energy of emitted PXR photon flux is changed with changing of the orientation angle between an emitting electron and reflecting crystallographic plane. The direction of this changing for the fixed observation angle $\Theta$ is opposite to that realizing in the process of PXR to Bragg scattering direction;

- To obtain an appropriate ratio signal/background it is necessary to collimate the total emission at the large observation angle $\Theta_{\|}$in the reaction plane. The value of such an angle can be determined by the Eqs. (30)-(32), (37) and the curves presented in Fig. 9;

- The total emission yield increases, but the ratio signal/background decreases with increasing of the photon collimator angular size $\Delta \Theta_{\|}$. Appropriate value of $\Delta \Theta_{\|}$depends on the concrete experimental conditions and can be determined by the Eq. (37) and Figs. 10 and 12;

- The forward PXR spectral width depends strongly on the target atomic number $Z$ and increases essentially with increasing of $Z$. Since this effect allows to increase the total emission yield and to reduce the requirements for X-ray detector energy resolution the heavy crystals are more appropriate as radiators for the forward PXR observation.

\section{Acknowledgements}

This work was supported in part by RFBR (grants: 02-02-16941 and 01-02-17471); Universities of Russia (grants: 02.01.012 and 02.01.018) and Integration (grant I-0751). One of the authors (A.K.) is grateful to CRDF (grant VZ-010-0)

\section{References}

[1] M.L. Ter-Mikaelian, High-Energy Electromagnetic Processes in Condensed Media, Wiley, New York, 1972.

[2] G.M. Garibian, C. Yang, Sov. Phys. JETP 34 (1972) 495.

[3] V.G. Baryshevsky, I.D. Feranchuk, Sov. Phys. JETP 34 (1972) 502.
[4] K.-H. Brenzinger, C. Herberg, B. Limburg, H. Backe, S. Dambach, H. Euteneuer, F. Hagenbuck, H. Hartman, K. Johann, K.-H. Kaiser, O. Kettig, G. Knies, G. Kube, W. Lauth, H. Schope, Th. Walcher, Z. Phys. A 358 (1997) 107.

[5] A. Caticha, Phys. Rev. B 45 (1992) 9541.

[6] Yu.N. Adischev, V.A. Versilov, A.P. Potylitsin, S.R. Uglov, S.A. Vorobiev, Nucl. Instr. and Meth. B 44 (1989) 130.

[7] O.V. Chefonov, B.N. Kalinin, G.A. Naumenko, D.V. Padalko, A.P. Potylitsin, I.E. Vnukov, I. Endo, M. Inoue, Nucl. Instr. and Meth. B 173 (2001) 18.

[8] I.D. Feranchuk, A.I. Ivashin, J. Phys. 46 (1985) 1981.

[9] V.G. Baryshevsky, I.D. Feranchuk, Nucl. Instr. and Meth. A 228 (1985) 490.

[10] N.N. Nasonov, A.G. Safronov, in: Yu.L. Pivovarov, A.P. Potylitsin (Eds.), Proceedings of the International Symposium on Radiation of Relativistic Electrons in Periodical Structures, Tomsk, 1993.

[11] G.M. Garibian, C. Yang, JETP 63 (1972) 1198.

[12] V.G. Baryshevsky, I.D. Feranchuk, Phys. Lett. A 57 (1976) 183.

[13] V.G. Baryshevsky, Nucl. Instr. and Meth. B 122 (1997) 13.

[14] C.L. Yuan Luke, P.W. Alley, A. Bamberger, G.F. Dell, H. Uto, Nucl. Instr. and Meth. A 234 (1985) 426.

[15] B.N. Kalinin, G.A. Naumenko, D.V. Padalko, A.P. Potylitsin, I.E. Vnukov, Nucl. Instr. and Meth. B 173 (2001) 253.

[16] G. Kube, C. Ay, H. Backe, N. Clawiter, M. Ghraly, F. Hagenbuck, K.-H. Kaiser, O. Kethig, W. Lauth, H. Mannweiler, D. Schroff, Th. Walcher, T. Weber, Abstract V International Symposium on Radiation from Relativistic Electrons in Periodic Structures, 10-14 September 2001, Lake Aya, Altai Mountains, Russia.

[17] N. Imanishi, N. Nasonov, K. Yajima, Nucl. Instr. and Meth. B 173 (2001) 227.

[18] N. Nasonov, in: H. Wiedemann (Ed.), Proceedings of the NATO-ARW Electron-Photon Interactions in Dense Media, Nor-Hamberg (Armenia) 2001, NATO Science Series II, Vol. 49, Kluwer Academic Publishers, 2002, p. 49.

[19] Z. Pinsker, Dynamical Scattering of X-rays in Crystals, Springer, Berlin, 1984.

[20] I.K. Bdikin et al., Tech. Phys. Lett. 25 (1999) 933.

[21] I.K. Bdikin, S.I. Bozhko, V.N. Semenov, I.A. Smirnova, V.G. Glebovski, S.N. Ermolov, V.Sh. Shekhtman, Poverhnost'. Rentgenovskie, sinhrotronnye i neitronnye issledovaniya 3 (2001) 15 (in Russian).

[22] I.E. Vnukov, B.N. Kalinin, A.A. Kiryakov, G.A. Naumenko, D.V. Padalko, A.P. Potylitsyn, Russ. Phys. J. 44 (2001) 281.

[23] A.P. Antipenko, G.L. Bochek, S.V. Blazhevich, et al., Nucl. Instr. and Meth. B 48 (1990) 291.

[24] E. Bogomazova, V. Boiko, S. Bozhko, B. Kalinin, G. Naumenko, D. Padalko, A. Potylitsyn, V. Semenov, A. Taratin, I. Vnukov, Abstract V International Symposium on Radiation from Relativistic Electrons in Periodic Structures, 10-14 September 2001, Lake Aya, Altai Mountains, Russia. 